Recepción: 27/09/16

Evaluación: 28/09/16

Aprobación: 29/11/16

Artículo de Investigación Científica

\title{
FACTORES ASOCIADOS AL LOGRO ACADÉMICO EN REGIONES DESARROLLADAS Y EN DESARROLLO DE COLOMBIA: UNA APROXIMACIÓN A PARTIR DE UN MODELO DE COMBINACIÓN DE CORTE TRANSVERSAL
}

\author{
Oscar Hernán Cerquera Losada* \\ Universidad Surcolombiana \\ Grupo De Investigación: Iguaque \\ oscar.cerquera@usco.edu.co \\ Julio Roberto Cano Barrera** \\ Universidad Surcolombiana \\ Grupo De Investigación: Iguaque \\ cafagose@hotmail.com
Camilo Fabiám Gómez Segura***
Universidad Surcolombiana Grupo De Investigación: Iguaque julio.cano@usco.edu.co

\begin{abstract}
RESUMEN
En este trabajo, se analizan los determinantes del rendimiento académico de la educación media, al comparar los resultados en el área de matemáticas de la Prueba Saber $11^{\circ}$ de los estudiantes de grado once de las regiones con mayor y menor nivel de desarrollo socioeconómico de Colombia. La metodología utilizada se desarrolló a través de la aplicación de un Modelo
\end{abstract}

* Economista de la Universidad Surcolombiana, Magister en Economía por la Universidad de Buenos Aires. Docente Investigador de la Universidad Surcolombiana. Joven Investigador e Innovador de Colciencias 2010-2011; Investigador del Observatorio Regional del Mercado de Trabajo del Huila, ORMET Huila. Integrante del grupo de investigación Iguaque.

** Administrador de Empresas de la Universidad Surcolombiana, especialista en Gerencia Social y en Gestión del Desarrollo Regional, Magister en Investigación Operativa y Estadística de la Universidad Tecnológica de Pereira. Docente Investigador de la Universidad Surcolombiana. Integrante del grupo de investigación Iguaque.

*** Economista de la Universidad Surcolombiana, Magister en Economía de la Universidad Nacional de la Plata. Docente Investigador de la Universidad Surcolombiana. Investigador del Observatorio Regional del Mercado de Trabajo del Huila, ORMET Huila. Integrante del grupo de investigación Iguaque. 
de Combinación de Corte Transversal para el periodo de estudio de 2008 a 2013. Los resultados muestran que existen importantes diferencias entre los determinantes del rendimiento académico en regiones con mayor y menor nivel de desarrollo socioeconómico, las características personas de los estudiantes, tales como la edad y la etnia, así como las características de las escuelas tienen mayor impacto sobre los estudiantes de las regiones con menor nivel de desarrollo, mientras que las características familiares y del hogar, tales como educación de los padres, disponer de computador en casa y los ingresos familiares, presentan un mayor impacto sobre el rendimiento académico de los estudiantes de regiones con mayor nivel de desarrollo socioeconómico.

Palabras Clave: Rendimiento académico, Prueba Saber $11^{\circ}$, combinación de Corte Transversal, regiones con mayor nivel de desarrollo socioeconómico, regiones con menor nivel de desarrollo socioeconómico.

\title{
FACTORS ASSOCIATED WITH ACADEMIC ACHIEVEMENT IN DEVELOPED AND DEVELOPING REGIONS OF COLOMBIA: AN APPROACH FROM A CROSS-SECTION COMBINATION MODEL
}

\begin{abstract}
In this paper, we analyze the determinants of the academic performance of secondary education, by comparing the results in the area of mathematics from the Saber 11 test of eleventh grade students in the regions with higher and lower levels of socioeconomic development in Colombia. The methodology used was developed through the application of a Cross-Section Combination Model for the study period from 2008 to 2013. The results show that there are important differences between the determinants of academic performance in regions with higher and lower levels of socioeconomic development, the personal characteristics of students, such as age and ethnicity, as well as the characteristics of schools which have a greater impact on students in regions with lower levels of development, whereas family and home characteristics such as parental education, the use of a home computer and family income, have a greater impact on the academic performance of students from regions with higher levels of socioeconomic development.
\end{abstract}

Key words: Academic performance, Saber test 11th, cross section combination model, regions with higher level of socioeconomic development, regions with lower level of socioeconomic development. 


\title{
FATORES ASSOCIADOS À REALIZAÇÃO ACADÊMICA EM REGIÕES DESENVOLVIDAS E DESENVOLVIMENTO DA COLÔMBIA: UMA APROXIMAÇÃO DE UM MODELO DE COMBINAÇÃO DE CORTE TRANSVERSAL
}

\begin{abstract}
RESUMO
Neste trabalho, analisamos os determinantes do desempenho acadêmico do ensino secundário, comparando os resultados na área de matemática do teste Saber 11 dos alunos da décima primeira série das regiões com maior e menor nível de desenvolvimento socioeconômico da Colômbia . A metodologia utilizada foi desenvolvida através da aplicação de um modelo de combinação transversal para o período de estudo de 2008 a 2013. Os resultados mostram que há diferenças importantes entre os determinantes do desempenho acadêmico em regiões com níveis de desenvolvimento mais altos e mais baixos As características socioeconómicas., as características particulares dos estudantes como idade e etnia, assim como as características das escolas têm maior impacto nos estudantes em regiões com menor nível de desenvolvimento, enquanto as características familiares e domésticas, como educação de pais, computador doméstico e renda familiar, têm um impacto maior no desempenho acadêmico de estudantes de regiões com maiores níveis de desenvolvimento socioeconômico.
\end{abstract}

Palavras-chave: desempenho acadêmico, teste Saber $11^{\circ}$, combinação de Corte transversal, regiões com maior nível de desenvolvimento socioeconômico, regiões com menor nível de desenvolvimento socioeconômico.

\section{INTRODUCCIÓN}

La educación siempre se la ha considerado como algo que ejerce fuertes poderes transformadores. Los gobiernos la consideran como una ruta hacia la independencia y la formación de la ciudadanía; los sociólogos la ven como un motor de transformación social y de mayor conciencia en las clases "oprimidas"; los economistas la ven como un elemento importante, que aumenta e iguala los ingresos y las oportunidades de las personas (Vegas y Petrow, 2008). La educación es un tema que despierta el interés de toda una sociedad, no solo por el efecto que tiene sobre el crecimiento y desarrollo de las economías, sino, también, porque permite un mayor nivel de desarrollo personal.

En este trabajo, se examinan los determinantes del rendimiento académico en matemáticas, al analizar a los estudiantes de grado once de las regiones con mayor y menor nivel de desarrollo socioeconómico de Colombia. El rendimiento académico se evalúa a través de la prueba Saber $11^{\circ}$ de matemáticas, y las características económicas, sociales, y culturales de los individuos, el hogar, y la institución educativa, se consideran como los factores o variables que explican un 
mayor o menor rendimiento académico. Se utilizaron los datos de los alumnos de grado once, o último año de bachillerato, de instituciones educativas de Bogotá, Antioquía y Santander, en representación de las regiones con mayor nivel de desarrollo, y Chocó, Sucre y Vichada, en representación de las regiones con menor nivel de desarrollo.

Para resolver el problema propuesto, se utilizó el enfoque teórico de la función de producción educativa que propuso inicialmente Hanusheck (1986), donde el resultado académico representa el producto obtenido a partir de la combinación de un grupo de factores productivos, representados por las características del estudiante, la familia y la escuela.

Este enfoque teórico se desarrolló a través de la aplicación de un Modelo de Combinación de Corte Transversal, que permite analizar combinaciones de unidades de estudio temporal con unidades de corte transversal para el periodo de estudio de 2008-2013.

Por esto, con este estudio se busca resolver la siguiente pregunta: ¿Cuáles son los factores que determinan el rendimiento académico de la educación media en matemáticas, en Colombia, al comparar regiones de contextos socioeconómicos favorables y regiones con contextos socioeconómicos menos favorables, durante el periodo 2008-2013?

Este documento se divide en seis apartados: el primero contiene la introducción, el segundo se orienta al planteamiento del problema, el tercero recopila el referente teórico, el cuarto se centra en la metodología aplicada, el quinto expone los resultados obtenidos y el último presenta las principales conclusiones del trabajo.

\section{PLANTEAMIENTO DEL PROBLEMA}

Varios estudios han abordado el problema del rendimiento académico y la eficiencia del sector educativo; ejemplo de ello, Heinesen y Graversen (2005) y Hanushek (1986, 1989). Dentro de los factores que afectan el rendimiento académico se han destacado las condiciones socioeconómicas de los alumnos, las características físicas de los planteles educativos, la formación de los docentes, sus esquemas de remuneración e incentivos, entre otros. En el caso colombiano, se han realizado diversos trabajos que analizan la calidad de la educación y sus determinantes: Piñeros y Rodríguez (1998); Gaviria y Barrientos (2001); Núñez, Steiner, Cadena y Pardo (2002); Barrera y Gaviria (2003) y Mina, (2004).

Según estadísticas del Departamento Administrativo Nacional de Estadística (DANE), para el año 2013, los indicadores revelan grandes diferencias económicas y sociales en cada uno de los Departamentos de Colombia; ejemplo de ello el Producto Interno Bruto per cápita, que establece que regiones como Bogotá (22.735.038), y los Departamentos de Santander (25.545.682) y Antioquia (14.675.420), se destacan, mientras que Departamentos como Chocó (6.210.268), Sucre (6.675.953) y Vichada (5.876.777) están siempre por debajo del promedio nacional. 


\section{Gráfica 1. Comportamiento del PIB per cápita para las regiones desarrolladas y menos desarrolladas en Colombia durante el periodo 2008-2013.}

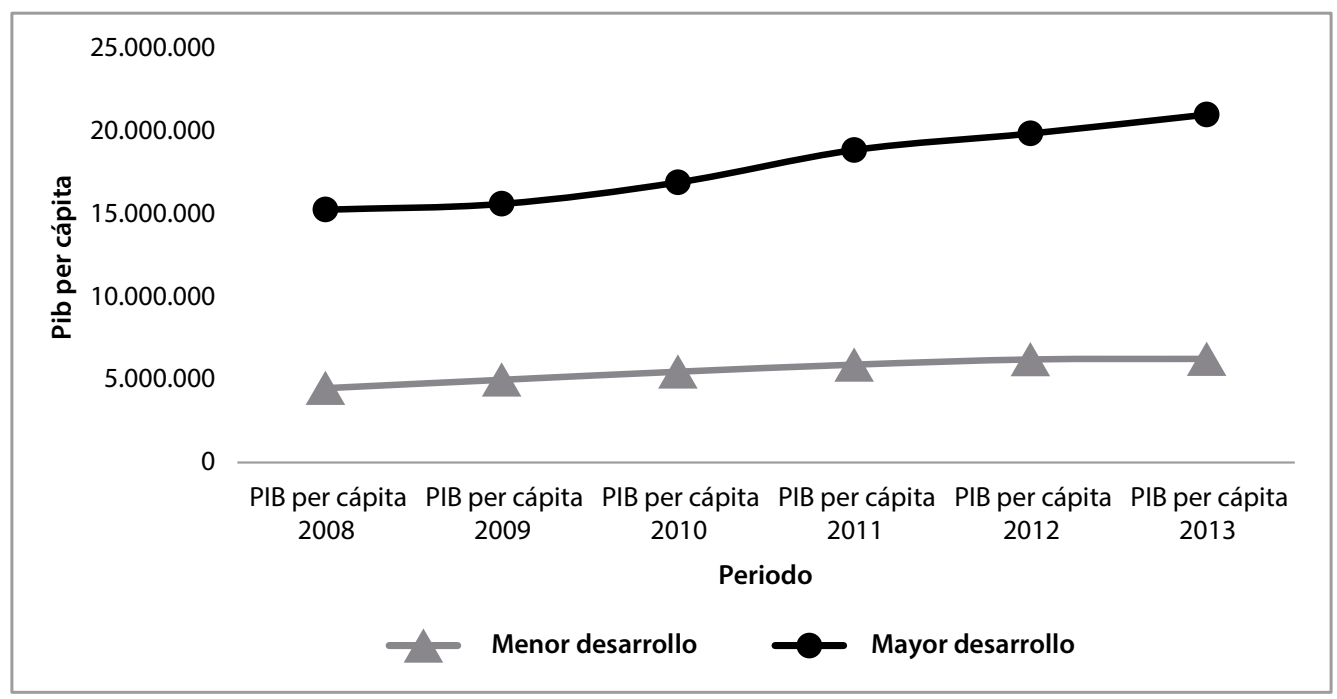

Fuente: Esta investigación, con base en información del Departamento Administrativo Nacional de Estadística.

El Índice de Necesidades Básicas Insatisfechas (NBI) muestra las desigualdades sociales en las regiones de Colombia; los datos confirman que las regiones con mayor desarrollo presentan niveles bajos de NBI: Bogotá $(10,71 \%)$, Santander $(15,19 \%)$ y Antioquia (18,29\%); caso contrario ocurre en Chocó (87,57\%), Sucre $(63,70 \%)$ y Vichada (45,58\%). Del mismo modo, al analizar la tasa de incidencia de la pobreza, se encuentra que es mayor en los Departamentos que presentan un menor nivel de desarrollo socioeconómico, Chocó (68\%) y Sucre (51,5\%), y sobresalen las regiones con mayor nivel de desarrollo, Bogotá $(11,6 \%)$, Santander $(20,8 \%)$ y Antioquia $(26,8 \%)$, con una menor tasa de pobreza.

Con respecto a la cobertura en el número instituciones educativas públicas y privadas, en los Departamentos con mayor nivel de desarrollo socioeconómico, existe un gran número de instituciones educativas: Bogotá cuenta con 983 colegios privados y 697 públicos; en Santander existen 302 privados y 510 públicos, y Antioquia dispone de 522 privados y 1105 públicos. En las regiones con menor nivel de desarrollo, Chocó (7 privados y 190 públicos), Sucre (67 privados y 232 públicos) y Vichada (0 privados y 18 públicos); de modo que la presencia de establecimientos educativos públicos y privados es relativamente menor en estas regiones, en comparación con las regiones de mayor desarrollo. 


\section{Gráfica 2. Puntaje Promedio de Matemáticas para las regiones desarrolladas y menos desarrolladas en Colombia durante el periodo 2000-2013.}

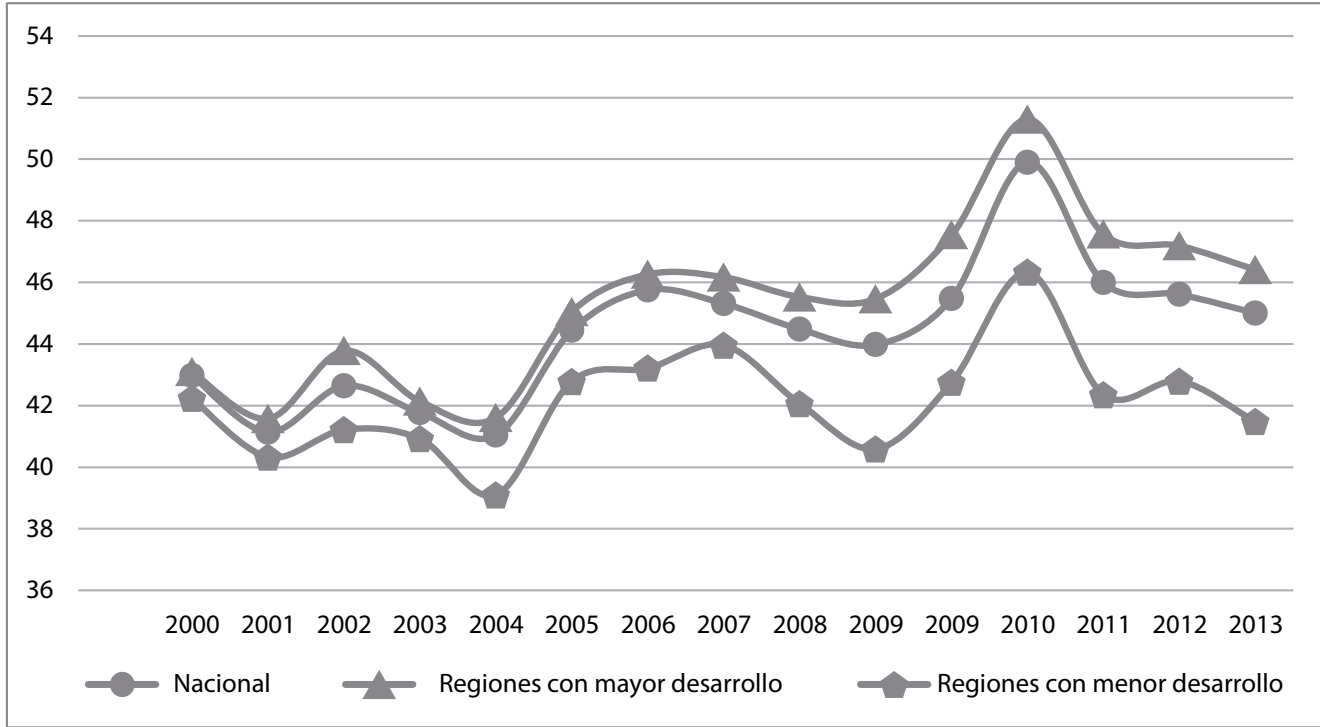

Fuente: Esta investigación, con base en información del Instituto Colombiano para la Evaluación de la Educación Superior.

La Gráfica 2 evidencia el puntaje promedio en matemáticas de las regiones con mayor nivel de desarrollo, respecto al puntaje promedio de las regiones con menor nivel de desarrollo. Para todo el periodo de estudio, el puntaje promedio de las regiones con mayor nivel de desarrollo $(45,3)$ se ubicó por encima de la media nacional $(44,32)$, y del promedio de las regiones con menor nivel de desarrollo $(42,18)$. Estos resultados muestran dos aspectos:

1) Los puntajes promedios empezaron el periodo con puntajes relativamente bajos, por lo menos hasta el 2004; de ahí en adelante, tendieron a aumentar hasta llegar a su punto más alto en 2010; a partir de allí, muestran una tendencia a la baja, con una leve aparente estabilización después del 2011.

2) Los puntajes promedio de los Departamentos con mayor nivel de desarrollo y el promedio nacional, se alejan cada vez más del puntaje promedio de los Departamentos con menores niveles de desarrollo; es decir, las diferencias entre los puntajes considerados se acrecentaron, lo que empeora la situación para las regiones de menor desarrollo.

Al analizar la situación descrita, se puede indagar sobre la relación positiva existente entre el desarrollo y crecimiento económico y social, respecto al desempeño académico presentado por las regiones. Las regiones con mejores indicadores económicos y sociales muestran mayores niveles de rendimiento 
académico, mientras que las regiones con indicadores socioeconómicos más bajos presentan menores niveles de desempeño académico. No cabe duda que el entorno socioeconómico en el que vive el estudiante afecta su rendimiento académico; este grupo de regiones presenta características muy diferentes; por lo tanto, los factores que determinan un mejor rendimiento académico deben ser diferentes.

El interés por investigar esta situación va más allá de las inquietudes por lo desconocido, pues, desde el punto de la política pública, normalmente se toman decisiones que se aplican de igual forma a todas las regiones del país, sin reconocer que hay lugares con características diferentes, donde muy seguramente dichas decisiones de políticas no tendrán el efecto esperado.

$\mathrm{Al}$ conocer el contexto socioeconómico de las regiones que presentan un alto desarrollo (Bogotá, Santander y Antioquia), respecto de las de menor desarrollo (Sucre Vichada y Chocó), el objetivo de esta investigación es determinar los factores que influyen en el rendimiento académico de la educación media en matemáticas, en Colombia, al comparar regiones de mayor y menor nivel de desarrollo socioeconómico, durante el periodo 2008-2013.

\section{REFERENTE TEÓRICO}

\subsection{Antecedentes}

El rendimiento académico de un estudiante puede depender de una cantidad de factores que, en algunos casos, presenta cierta relación entre sí, pero que, en otros, no se relacionan en lo absoluto. Estos factores van desde la educación de los padres y los valores de la sociedad, hasta la infraestructura escolar y otra serie de factores inimaginable (Vegas y Petrow, 2008). Generalmente, en la literatura, estos factores pueden agruparse en tres categorías: factores referentes a los estudiantes, factores referentes al colegio y factores referentes al sistema, que interactúan en un contexto económico, político y social.

\section{Características de los estudiantes}

Coleman (1966) es uno de los primeros investigadores en estudiar el efecto de las características del estudiante sobre el rendimiento académico; en sus estudios sobre calidad educativa, encontró que las condiciones socioeconómicas de los estudiantes y sus antecedentes familiares causan los mayores efectos sobre el rendimiento académico.

Según Vegas y Petrow (2008), la edad a la que los estudiantes ingresan a la educación primaria puede afectar la trayectoria del niño en el Sistema educativo y sus logros académicos, pero esta correlación difiere de acuerdo con el tipo de países de donde proviniera el estudiante.

Angrist y Krueger (1991) encontraron que la entrada retardada a la educación se relaciona con un menor logro educativo, evidenciado especialmente a través del abandono de los estudios de forma prematura, pues, al llegar a la edad promedio de terminación de estudios hay una deserción, sin haberlos culminado. Fuchs 
y Woessmann (2005) encuentran que, especialmente en Colombia, existe una correlación positiva entre la situación socioeconómica y el rendimiento educativo de los estudiantes de primaria.

Gaviria y Barrientos (2001) estudian los efectos del entorno familiar y las características del plantel sobre el rendimiento escolar en Bogotá y encuentran que la calidad de la educación secundaria es un factor clave en el logro socioeconómico de las personas y, por lo tanto, un factor determinante en el acceso a las oportunidades y la movilidad social.

Por último, Cerquera (2011), al aplicar un modelo multinivel con información de las pruebas Saber $11^{\circ}$, determina los factores del rendimiento académico en Neiva; este autor encuentra que los estudiantes con computador en casa alcanzan un rendimiento mayor que quienes no tienen computador en casa.

\section{Características de la Escuela}

Según Sanders y Rivers (1996), las características y el comportamiento del personal docente determinan, de manera considerable, el aprendizaje estudiantil del alumno. Un docente ineficaz reduce potencialmente el desempeño de un estudiante durante años.

Darling (2000), al utilizar datos de la Encuesta de Política para todos los Estados Unidos, de las encuestas de personal docente, y de la evaluación nacional de progreso educativo para los años 1993-1994, examinó la relación entre las características del docente y otros insumos de la escuela, respecto al rendimiento académico de los estudiantes.

Según Hepp K., Hinostroza S., Laval M., y Rehbein F. (2004), la inversión en TIC's en la educación se considera como la principal forma de preparación de las personas para las nuevas necesidades que demanda el mercado laboral y permitir que regiones rezagadas desarrollen las competencias necesarias para igualar las oportunidades de acceso al empleo.

Piñeros y Rodríguez (1999) encuentran que "las escuelas tienen un efecto pequeño pero considerable sobre el logro académico, teniendo entre 15\% y $18 \%$ de la varianza entre estudiantes en escuelas privadas y $12 \%$ y $16 \%$ en escuelas públicas".

Caro (2000) encuentra que, en los estudiantes de $3^{\circ}$ y $5^{\circ}$ de Bogotá, el nivel de escolaridad de los profesores se relaciona con el rendimiento de los estudiantes. Entre tanto, Fuchs y Woessmann (2005), al utilizar el PIRLS de 2001, sostiene que no hay ninguna relación estadísticamente significativa entre el desempeño académico de los estudiantes y el nivel académico de los docentes, en Colombia.

Chica, Galvis, y Ramírez (2010) encontraron que los estudiantes de jornada completa obtienen puntajes más altos en comparación con los estudiantes que asisten a otras jornadas, como las nocturnas, sabatinas y dominicales. 
López (2010), a partir de las pruebas Saber 11², para el periodo 1980-2009, estima el porcentaje de la variabilidad del logro académico atribuible al plantel educativo dentro de un análisis multinivel discriminado por sector educativo.

Barrientos (2008) analiza la calidad de la educación pública y el logro académico con las pruebas ICFES 2004-2006 en Medellín, a través de un modelo de aproximación por regresión intercuartil.

\section{Características organizacionales y políticas}

Fuchs y Woessmann (2005) sugieren que los factores organizativos pueden explicar hasta un $25 \%$ de las variaciones en los puntajes entre los países; la mayor autonomía escolar en la administración del personal y las decisiones sobre procesos (contratación de docentes, selección de textos, asignaciones presupuestales dentro de los colegios) se correlacionan fuertemente con un mejor desempeño estudiantil.

King y Ozler (2000), al utilizar una función de producción educativa, evalúan el impacto que tuvo la reforma de la autonomía escolar, en Nicaragua, sobre el aprendizaje estudiantil.

Filmer y Eskeland (2002) encuentran resultados similares para estudiantes de $6^{\circ}$ y $7^{\circ}$ de Argentina, donde la autonomía de los colegios de primaria se asocia con un mejor desempeño estudiantil.

Según Wolf, Navarro y González (2005), América Latina presenta grandes antecedentes de provisión privada de escolaridad, de manera más consistente en los niveles de educación preescolar, secundaria y superior.

Cox y Jiménez (1991) examinan la eficiencia relativa de las escuelas públicas y privadas de Colombia y Tanzania; es decir, estudian el rendimiento de ambos sectores al comparar entre los dos países; encuentran que, en ambos países, los estudiantes de escuelas privadas tienen calificaciones mayores que los estudiantes de escuelas públicas.

Piñeros y Rodríguez (1999) encuentran que los estudiantes de las escuelas públicas rinden mejor que los estudiantes de las escuelas privadas. Como evidencia los resultados de estos estudios, la situación socioeconómica de los estudiantes juega un papel preponderante en la determinación del desempeño de los estudiantes de las escuelas tanto privadas como públicas.

Mina (2004), en su investigación "Factores Asociados al Logro Educativo a Nivel Municipal", estudia las variables que inciden en la calidad de la educación en 897 municipios de Colombia durante el periodo 1966-1999, a través de la estimación de una función de producción en educación con una estructura de panel de datos. Mina encuentra que el gasto en educación por niño (positivo), la pobreza y la desigualdad (negativo) de cada municipio se correlacionan significativamente con el rendimiento académico. 


\subsection{Marco Teórico}

Hanushek (1986), Ph.D. en Economía del Instituto de Tecnología de Massachusetts (MIT), actual profesor de la Universidad de Stanford, reconocido investigador en el campo de la educación, ha realizado importantes aportes para el análisis económico de la educación; él ha integrado la economía y la educación con mucho éxito a través de la función de producción educativa, al señalar que "la economía puede analizar la educación con un enfoque de insumo-producto, es decir, un marco en el cual la calidad de la educación es determinada por una serie de factores".

Una función de producción en educación se refiere al análisis estadístico que permite observar los resultados de los estudiantes ${ }^{\mathbf{1}}$ en función de un conjunto de características que pueden ser propias, como la edad, el sexo, características familiares, y de la escuela. El uso de la función de producción en el campo educativo se ha convertido en una herramienta potencial para el análisis y la definición de políticas en diferentes países del mundo.

El modelo conceptual generalmente aceptado para establecer la función de producción en educación tiene la siguiente especificación:

$R_{i t}=f\left(F_{i}^{(t)}, P_{i}^{(t)}, O_{i}^{(t)}, I_{i}\right) R_{i t}=f\left(F_{i}^{(t)}, P_{i}^{(t)}, O_{i}^{(t)}, I_{i}\right)$

Donde, $i i$ representa el i-ésimo estudiante y $t t$ el periodo de tiempo,

$R_{i,} R_{i+}=$ resultado escolar del estudiante $i i$ en el tiempo $t t$

$F_{i}^{(t)} F_{i}^{(t)}=$ vector de características familiares en el tiempo $t t$

$P_{i}^{(t)} P_{i}^{(t)}=$ vector de compañeros de aula en el tiempo $t t$

$O_{i}^{(t)} O_{i}^{(t)}=$ vector de insumos escolares en el tiempo $t t$

$I_{i} I_{i}=$ vector de habilidades innatas del estudiante $i i$

La estructura funcional de la función de producción educativa relaciona, por un lado, los resultados escolares (rendimiento académico) a nivel individual por estudiante con un grupo de variables o insumos que caracterizan al estudiante, con los que se establece una relación teórica directa, tales como los insumos escolares (infraestructura, docentes), los insumos familiares y habilidades innatas.

Por simplicidad, y especialmente por la escasa disponibilidad de datos, en esta investigación el vector de compañeros de aula no se interpreta como un vector de variables aparte. Por otro lado, en el vector de habilidades innatas, se consideraron las características individuales del estudiante, más que habilidades altamente diferenciadoras entre individuos, porque en la práctica es difícil contar con mediciones iniciales de coeficiente intelectual que pudieran aplicarse a toda la población de estudio² .

1. Estos resultados se derivan, por lo general, de las pruebas estandarizadas aplicadas a los estudiantes.

2. Este es un problema común en las investigaciones de este tipo, dada la dificultad en la consecución de los datos. 


\section{DISEÑO METODOLÓGICO}

Como el propósito de esta investigación es identificar los factores escolares e individuales que determinan el rendimiento académico de los estudiantes en el área de matemáticas, se utilizó la base de datos suministrada por el ICFES, que recoge información de carácter personal, familiar y económico de los estudiantes que presentaron las pruebas Saber $11^{\circ}$ durante el periodo de estudio especificado, así como un conjunto de características básicas de la institución académica a la que pertenece el estudiante; además, el ICFES suministra el resultado en la prueba del estudiante, por áreas.

Una vez organizada la base de datos, se caracterizó cada una de las variables estudiadas a través del uso de la estadística descriptiva; de esta forma, se posibilitó obtener un análisis más detallado de la población objeto de estudio. Posteriormente, se desarrolló el modelo econométrico propuesto, una combinación de corte transversal, que permite analizar combinaciones de unidades de estudio temporal con unidades de corte transversal para el periodo de estudio 2008-2013. Se determinaron los factores del rendimiento académico para las regiones con mayor y menor nivel de desarrollo socioeconómico y se realizaron combinaciones entre ellas; además, se estimaron modelos a nivel departamental.

El estudio propuesto se ajusta a los propósitos de la investigación no experimental, "también conocida como investigación ex post facto, término que proviene del latín y significa después de ocurridos los hechos". De acuerdo con Kerlinger, la investigación no experimental es un tipo de investigación sistemática en el que el investigador no tiene control sobre las variables independientes porque ya ocurrieron los hechos o porque son intrínsecamente manipulables (Kerlinger, 1987, p. 269).

Dentro de la investigación, se seleccionó a todos los individuos que presentaron las Pruebas Saber $11^{\circ}$ y que cumplieran con las siguientes características:

Estudiantes habitantes de los Departamentos de Antioquia, Santander, Bogotá, Chocó, Sucre y Vichada.

Estudiantes activos del grado once durante los años 2008-2013.

Estudiantes activos que presentaron las Pruebas Saber $11^{\circ}$ y que, durante ese periodo, cursaban grado 11 . Se seleccionaron los estudiantes que cursaron el grado once y presentaron las pruebas durante el segundo semestre del año, para el periodo 2008-2013 ${ }^{3}$.

Estudiantes que asistieron a las dos sesiones; es decir, al examen completo.

Estudiantes que registran una calificación mayor o igual a 10 puntos en la prueba de matemáticas.

3. Se debe tener en cuenta que las Pruebas Saber $11^{\circ}$ las aplica el ICFES de manera semestral; para el segundo semestre del año, suele presentarse la gran mayoría de estudiantes, pues allí el estudiante representa a la institución educativa a la que pertenece. Debido a esto, se analizaron solo aquellos estudiantes que presentaron la prueba en el segundo semestre del año. 
Se trabajó con una muestra total de 1.222.116 datos, donde el 45,11\% de los casos corresponde a estudiantes de Bogotá, el 35\% de Antioquia y el 12,51\% de Santander, con respecto a la región con mayor nivel de desarrollo. Por otro lado, el 5,25\% de los estudiantes que conforman la muestra total residen en el Departamento de Sucre, el 1,92\% en el Chocó y el 0,21\% en Vichada.

\section{RESULTADOS}

\subsection{Análisis Estadístico Descriptivo}

Este análisis permite caracterizar la muestra objeto de estudio, al establecer el rendimiento promedio en matemáticas de los estudiantes en función de sus características generales, que incluyen características personales, del hogar y el colegio. Así mismo, en el Anexo 1 se incluyen las estadísticas de cada variable utilizada en la investigación.

La Tabla 1 muestra el puntaje promedio de matemáticas para las regiones con mayor y menor nivel de desarrollo socioeconómico durante el periodo 2008-2013. Como se puede observar, las regiones con mayor nivel de desarrollo registraron un $9 \%(4,24$ puntos) más de puntaje en relación con las regiones de menor nivel de desarrollo, con Bogotá (47,52 puntos) y Chocó (39,76 puntos), como la zona y Departamento que registran el mayor y menor puntaje, respectivamente.

Para analizar la desigualdad del puntaje entre regiones, se generan tres rangos intercuantílicos:4 90-10, 90-50 y 50-10. El rango intercuantílico 9010 corresponde al intervalo entre el $10 \%$ de los mayores y menores puntajes obtenidos por los estudiantes; es decir, es una medida que puede resumir la dispersión en la distribución del rendimiento de matemáticas. Como se puede observar, las regiones con mayor nivel de desarrollo socioeconómico, con un rango de 26 puntos, son más desiguales. Por su parte, respecto al rango intercuantílico 90-50, que resume la distribución en el rendimiento de matemáticas del $10 \%$ de los estudiantes con mejores puntajes en relación con la media, las estimaciones muestran que nuevamente las regiones con mayor nivel de desarrollo socioeconómico, con un rango de 14,82 puntos, son más desiguales para la población con mejores puntajes.

Sin embargo, todo lo contrario sucede para el rango intercuantílico 50-10, ya que, en este caso, las regiones con menor nivel de desarrollo, con un valor de 11,2 puntos, presentan una mayor desigualdad. Estas estimaciones muestran que al analizar la mayor parte de la distribución (rango intercuantílico 90-10), existe mayor desigualdad en las regiones desarrolladas, lo que puede deberse principalmente a que más del $90 \%$ de la población en las regiones con menor nivel de desarrollo pertenecen al estrato 1 y 2; es decir, tienen condiciones socioeconómicas similares.

4. Se considera la diferencia entre cuartiles. 
Tabla 1. Puntaje promedio de matemáticas para las regiones con mayor y menor nivel de desarrollo socioeconómico durante el periodo 2008-2013.

\begin{tabular}{|l|cccc|cccc|}
\hline & \multicolumn{3}{|c|}{ Región con mayor nivel de desarrollo } & \multicolumn{3}{c|}{ Región con menor nivel de desarrollo } \\
\cline { 2 - 9 } & Antioquia & Bogotá & Santander & Total & Chocó & Sucre & Vichada & Total \\
\hline Puntaje Matemáticas & 44,70 & 47,52 & 46,99 & 46,38 & 39,76 & 43,03 & 41,81 & 42,14 \\
Quantil 10 & 33,00 & 35,00 & 34,00 & 34,00 & 29,12 & 31,00 & 30,84 & 30,80 \\
Quantil 50 & 44,00 & 46,62 & 46,00 & 45,18 & 39,55 & 42,39 & 42,00 & 42,00 \\
Quantil 90 & 57,88 & 61,00 & 61,00 & 60,00 & 49,84 & 55,00 & 53,00 & 54,00 \\
R, intercuantílico (90-10) & 24,88 & 26,00 & 27,00 & 26,00 & 20,72 & 24,00 & 22,16 & 23,20 \\
R, intercuantílico (90-50) & 13,88 & 14,38 & 15,00 & 14,82 & 10,29 & 12,61 & 11,00 & 12,00 \\
R, intercuantílico (50-10) & 11,00 & 11,62 & 12,00 & 11,18 & 10,43 & 11,39 & 11,16 & 11,20 \\
\hline
\end{tabular}

Fuente: Esta investigación, con base en información de Instituto Colombiano para el Fomento de la Educación Superior.

La Tabla 2 presenta el puntaje promedio de matemáticas, según las características del estudiante para las regiones con mayor y menor nivel de desarrollo socioeconómico durante el periodo 2008-2013. Se observa una clara diferencia en cada uno de sus componentes; los estudiantes varones obtienen un puntaje en matemáticas mayor que las mujeres, que es mayor en las regiones con mayor nivel de desarrollo socioeconómico (5,16 puntos), frente a las regiones con menor nivel de desarrollo (3,52 puntos). En relación con el rendimiento promedio de matemática y la edad, se observa que, a medida que los estudiantes incrementan su edad, el puntaje promedio disminuye para las dos regiones evaluadas. La diferencia en el puntaje promedio entre las regiones con mayor y menor nivel de desarrollo socioeconómico se da a favor de las primeras y es mayor para los estudiantes de 16 y 17 años con una brecha de 9\% (4,14 puntos).

Tabla 2. Puntaje promedio de matemáticas, según las características del estudiante para las regiones con mayor y menor nivel de desarrollo durante el periodo 2008-2013.

\begin{tabular}{|c|c|c|c|c|c|c|c|c|}
\hline \multirow{2}{*}{$\begin{array}{l}\text { Características del } \\
\text { Estudiante }\end{array}$} & \multicolumn{4}{|c|}{ Región con mayor nivel de desarrollada } & \multicolumn{4}{|c|}{ Región con menor nivel de desarrollo } \\
\hline & Antioquia & Bogotá & Santander & Total & Chocó & Sucre & Vichada & Total \\
\hline \multicolumn{9}{|l|}{ Género } \\
\hline Hombre & 46,34 & 49,28 & 48,69 & 48,13 & 40,14 & 44,03 & 42,41 & 42,97 \\
\hline Mujer & 43,44 & 46,00 & 45,61 & 44,95 & 39,43 & 42,16 & 41,22 & 41,43 \\
\hline \multicolumn{9}{|l|}{ Edad } \\
\hline$>=15$ & 47,04 & 49,77 & 50,12 & 49,11 & 42,82 & 47,65 & 46,83 & 46,26 \\
\hline $16 \times 17$ & 46,13 & 48,37 & 48,31 & 47,55 & 40,60 & 44,26 & 42,93 & 43,41 \\
\hline $18 \times 19$ & 41,87 & 44,37 & 43,30 & 43,16 & 38,60 & 40,22 & 40,92 & 39,76 \\
\hline $20 \times 21$ & 39,77 & 41,74 & 41,11 & 40,61 & 37,78 & 38,94 & 38,48 & 38,49 \\
\hline 22 y 23 & 39,49 & 41,53 & 40,47 & 40,32 & 37,60 & 38,57 & 38,53 & 38,25 \\
\hline
\end{tabular}


Oscar Hernán Cerquera Losada - Julio Roberto Cano Barrera - Camilo Fabiám Gómez Segura

\begin{tabular}{|l|cccc|cccc|}
\hline \multicolumn{1}{|c|}{$\begin{array}{c}\text { Características del } \\
\text { Estudiante }\end{array}$} & \multicolumn{4}{c|}{ Región con mayor nivel de desarrollada } & \multicolumn{4}{c|}{ Región con menor nivel de desarrollo } \\
\cline { 2 - 9 } & Antioquiaa & Bogotá & Santander & Total & Chocó & Sucre & Vichada & Total \\
\hline 24 y 25 & 39,29 & 41,65 & 40,41 & 40,26 & 36,64 & 37,95 & 35,92 & 37,36 \\
$>=26$ & 38,59 & 40,64 & 39,44 & 39,47 & 36,77 & 37,94 & 38,11 & 37,62 \\
Etnia & & & & & & & & \\
Negro & 41,26 & 45,23 & 46,86 & 42,22 & 39,55 & 40,28 & 40,39 & 39,65 \\
Indígena & 40,08 & 45,13 & 45,57 & 43,06 & 35,67 & 42,07 & 38,61 & 41,60 \\
Raizal & 40,84 & 47,86 & 48,35 & 45,07 & 41,42 & 39,05 & - & 40,28 \\
Otras & 44,13 & 46,65 & 47,12 & 45,97 & 40,47 & 41,63 & 38,42 & 41,06 \\
Prueba SABER & & & & & & & & \\
Presentó una vez & 44,84 & 50,71 & 50,59 & 50,15 & 39,24 & 45,45 & 43,38 & 44,30 \\
Presentó más de 2 veces & 44,24 & 52,28 & 52,73 & 52,03 & 39,01 & 44,52 & 50,27 & 44,25 \\
No trabaja & 44,99 & 47,85 & 47,65 & 46,73 & 39,87 & 43,20 & 41,90 & 42,30 \\
Trabaja & 41,90 & 44,29 & 42,90 & 43,15 & 38,58 & 40,49 & 41,15 & 39,92 \\
\hline
\end{tabular}

Fuente: Esta investigación, con base en información de Instituto Colombiano para el Fomento de la Educación Superior.

En cuanto a la etnia, ser raizal ${ }^{5}$, o pertenecer a otras razas, genera una brecha en el puntaje, entre regiones con mayor y menor nivel de desarrollo, de 4,79 $(11 \%), y$ 4,91 puntos $(11 \%)$ respectivamente, en que se destaca para ambas etnias el Departamento de Santander con un puntaje de 48,35 en los raizales y 47,12 para otras etnias. Con respecto al número de veces que se presentó la prueba, los resultados establecen que la brecha entre las regiones con mayor y menor nivel de desarrollo socioeconómico aumenta a medida que se presenta más veces la prueba. Como se esperaba, los estudiantes que trabajan presentan un puntaje promedio menor que aquellos estudiantes que no trabajan. Al comparar este resultado para las regiones con mayor y menor nivel de desarrollo, se encuentra que existe una discrepancia de 4,43 y 3,23 puntos respectivamente, entre los estudiantes que no trabajan y los que desempeñan alguna actividad laboral.

La evidencia empírica muestra que las características del colegio son un factor fundamental a la hora de explicar el rendimiento educativo de los estudiantes. La Tabla 3 presenta el puntaje promedio de matemáticas, según las características del colegio en las regiones con mayor y menor nivel de desarrollo socioeconómico durante el periodo analizado. Como se puede observar, las instituciones educativas normalistas son el tipo de colegio que presenta un mayor rendimiento en matemáticas en las dos regiones; sin embargo, existe una brecha de 5,8 puntos en favor de las regiones desarrolladas.

Los estudiantes de colegios privados registraron un puntaje promedio en matemáticas de 48,88 y 43,69 para las regiones con mayor y menor nivel de desarrollo socioeconómico respectivamente. Del mismo modo, los alumnos

5. Cabe destacar que en el Departamento de Vichada no existe población de esta etnia. 
de instituciones educativas públicas obtuvieron mejores puntajes en regiones desarrolladas $(45,01)$ que en regiones con menor nivel de desarrollo $(41,95)$. Adicionalmente, se observa un mejor rendimiento promedio en matemáticas de los estudiantes de colegios privados con respecto a los estudiantes de colegios públicos.

Los estudiantes que pagan pensión obtienen un mayor puntaje en matemáticas; en las regiones con mayor nivel de desarrollo socioeconómico, los que pagan pensión obtienen 4,93 puntos más que los estudiantes que no pagan, mientras que en las regiones con menor nivel de desarrollo dicha diferencia corresponde a 2,15 puntos. Al analizar la diferencia en el puntaje de matemáticas por regiones, se encuentra que los estudiantes que pagaron pensión en las regiones desarrolladas obtienen 5,79 puntos más en relación con los estudiantes de las regiones con menor nivel de desarrollo.

Uno de los factores que afecta, en algunos casos, el rendimiento académico de los estudiantes es la jornada académica, debido a que el tiempo de los individuos para recibir conocimiento es mayor en la jornada completa comparada con las demás. Según los resultados obtenidos, los estudiantes de jornada completa, en las regiones con mayor y menor nivel de desarrollo socioeconómico, tienen un rendimiento en matemáticas adicional de 3,36 y 1,65 puntos respectivamente, al compararlo con la jornada de la mañana; 4,43 y 1,65 puntos en relación con la jornada tarde y 9,15 y 5,73 con respecto a la jornada nocturna y sabatina.

Tabla 3. Puntaje promedio de matemáticas, según las características del colegio para las regiones con mayor y menor nivel de desarrollo socioeconómico durante el periodo 2008-2013.

\begin{tabular}{|l|cccc|cccc|}
\hline \multirow{2}{*}{$\begin{array}{c}\text { Características } \\
\text { del Colegio }\end{array}$} & \multicolumn{3}{|c|}{ Región con mayor nivel de desarrollo } & \multicolumn{4}{c|}{ Región con menor nivel de desarrollo } \\
\cline { 2 - 9 } & Antioquia & Bogotá & Santander & Total & Chocó & Sucre & Vichada & Total \\
\hline Tipo de Colegio & & & & & & & & \\
Académico & 44,47 & 47,53 & 46,15 & 46,34 & 38,47 & 42,76 & 40,99 & 42,15 \\
Técnico & 45,09 & 47,49 & 47,60 & 46,44 & 40,32 & 43,67 & 41,71 & 42,15 \\
Normalista & 46,13 & 50,07 & 50,78 & 47,94 & 40,42 & 43,41 & 42,53 & 42,15 \\
Privado & 46,95 & 50,01 & 47,30 & 48,88 & 37,68 & 44,60 & 39,87 & 43,69 \\
Público & 43,90 & 45,48 & 46,89 & 45,01 & 39,89 & 42,79 & 41,82 & 41,95 \\
No paga pensión & 43,49 & 45,84 & 46,29 & 44,85 & 39,76 & 42,60 & 41,79 & 41,84 \\
Paga pensión & 49,98 & 49,86 & 48,97 & 49,78 & 39,83 & 45,88 & 41,87 & 43,99 \\
Jornada & & & & & & & & \\
Completa & 47,04 & 51,29 & 47,99 & 49,65 & 38,19 & 49,85 & 4,11 & 44,13 \\
Mañana & 45,44 & 46,38 & 47,88 & 46,29 & 40,16 & 43,46 & 41,95 & 42,48 \\
Tarde & 44,57 & 45,48 & 46,97 & 45,22 & 39,42 & 43,66 & 32,82 & 42,88 \\
Nocturna y sabatina & 39,62 & 41,73 & 40,74 & 40,50 & 37,72 & 38,59 & 41,41 & 38,39 \\
\hline
\end{tabular}

Fuente: Esta investigación, con base en información de Instituto Colombiano para el Fomento de la Educación Superior. 
La Tabla 4 muestra el puntaje promedio de matemáticas, según las características del hogar, para ambas regiones durante el periodo 2008-2013. Como se observa, los estudiantes de regiones desarrolladas que viven en la zona urbana y rural tienen un 5\% (2,10 puntos) y 13\% (6,02 puntos) más de puntaje en relación con los estudiantes de las regiones con menor nivel de desarrollo.

Tabla 4. Puntaje promedio de matemáticas, según las características del hogar para las regiones con mayor y menor nivel de desarrollo socioeconómico durante el periodo 2008-2013.

\begin{tabular}{|l|cccc|cccc|}
\hline \multicolumn{1}{|c|}{$\begin{array}{c}\text { Características del } \\
\text { Hogar }\end{array}$} & \multicolumn{6}{|c|}{ Región con mayor nivel de desarrollo } & \multicolumn{3}{c|}{ Región con menor nivel de desarrollo } \\
\cline { 2 - 9 } & Antioquia & Bogotá & Santander & Total & Chocó & Sucre & Vichada & Total \\
\hline Residencia urbana & 45,26 & 47,99 & 48,12 & 47,12 & 39,83 & 44,13 & 42,49 & 42,84 \\
Residencia rural & 43,50 & 46,26 & 44,92 & 44,89 & 39,61 & 41,37 & 40,67 & 40,98 \\
Educación del Padre & & & & & & & & \\
Ninguna & 42,36 & 45,48 & 44,75 & 43,53 & 38,87 & 40,92 & 40,73 & 40,33 \\
Primaria & 42,30 & 44,45 & 44,48 & 43,92 & 38,77 & 41,16 & 41,11 & 40,58 \\
Secundaria & 45,25 & 46,84 & 47,73 & 46,38 & 39,97 & 43,87 & 42,60 & 42,83 \\
Técnica & 49,73 & 50,84 & 51,80 & 50,62 & 41,65 & 46,96 & 43,58 & 45,65 \\
Superior & 52,90 & 53,95 & 55,16 & 53,79 & 42,88 & 49,61 & 45,21 & 47,33 \\
Educación de la Madre & & & & & & & & \\
Ninguna & 41,22 & 44,29 & 43,27 & 42,68 & 38,42 & 40,58 & 39,83 & 39,94 \\
Primaria & 41,92 & 44,07 & 44,05 & 43,14 & 38,64 & 41,11 & 40,50 & 40,50 \\
Secundaria & 44,96 & 46,76 & 47,59 & 46,20 & 39,95 & 43,88 & 43,24 & 42,84 \\
Técnica & 49,45 & 50,51 & 51,73 & 50,30 & 42,16 & 47,24 & 44,73 & 45,98 \\
Superior & 52,64 & 54,02 & 55,00 & 53,75 & 43,08 & 50,26 & 46,31 & 47,50 \\
Ocupación del Padre & & & & & & & & \\
Emprendedor & 47,35 & 49,37 & 48,58 & 48,69 & 40,41 & 44,26 & 42,93 & 43,43 \\
Directivo & 49,19 & 50,92 & 52,06 & 50,53 & 41,71 & 47,73 & 44,02 & 45,88 \\
Asalariado & 48,14 & 49,52 & 50,83 & 49,26 & 41,58 & 47,47 & 43,69 & 45,58 \\
Obrero & 44,26 & 45,72 & 46,08 & 45,17 & 40,39 & 43,74 & 42,07 & 42,78 \\
Independiente & 4,43 & 47,45 & 45,99 & 46,00 & 39,37 & 42,25 & 41,61 & 41,60 \\
Actividades del hogar & 42,96 & 44,99 & 44,47 & 44,04 & 39,27 & 40,72 & 38,57 & 40,21 \\
Otras & 43,19 & 46,18 & 46,57 & 44,84 & 39,40 & 42,60 & 40,85 & 41,47 \\
\hline
\end{tabular}

Fuente: Esta investigación, con base en información de Instituto Colombiano para el Fomento de la Educación Superior.

El nivel educativo de los padres es uno de los componentes más importantes a la hora de estudiar el rendimiento académico. Los estudiantes con padres que no tienen ningún nivel educativo, en las regiones con mayor y menor nivel de desarrollo socioeconómico, obtienen en promedio 10,26 y 7 puntos menos que los 
estudiantes con padres de nivel de Educación Superior; esta diferencia aumenta a medida que se compara con padres de mayor nivel educativo. En cuanto a la brecha del rendimiento en matemáticas por regiones, se observa que es positiva a favor de las regiones desarrolladas y, a su vez, creciente a medida que aumenta el nivel educativo de los padres; pasa de un diferencial de 7\% (3,20 puntos) para los estudiantes con padres sin ningún nivel educativo, a tener una discrepancia de $12 \%(6,46$ puntos $)$ para los alumnos cuyos padres tienen un nivel de Educación Superior.

Tabla 5. Puntaje promedio de matemáticas, según las características del hogar para las regiones con mayor y menor nivel de desarrollo socioeconómico durante el periodo 2008-2013.

\begin{tabular}{|l|cccc|cccc|}
\hline \multirow{2}{*}{ Estrato socioeconómico } & \multicolumn{5}{|c|}{ Región con mayor nivel de desarrollo } & \multicolumn{5}{c|}{ Región con menor nivel de desarrollo } \\
\cline { 2 - 9 } & Antioquia & Bogotá & Santander & Total & Chocó & Sucre & Vichada & Total \\
\hline Bajo & 42,82 & 45,38 & 45,12 & 44,31 & 39,65 & 42,63 & 41,85 & 41,80 \\
Medio & 47,34 & 50,02 & 50,62 & 49,21 & 45,40 & 49,17 & 45,90 & 46,33 \\
Alto & 55,79 & 55,44 & 57,42 & 55,83 & 39,92 & 50,99 & 39,99 & 48,66 \\
Ingresos & & & & & & & & \\
Menos de 1 SMMLV & 41,81 & 43,75 & 43,90 & 42,78 & 38,83 & 41,59 & 40,27 & 41,01 \\
Entre 1 y 2 SMMLV & 43,86 & 45,75 & 46,21 & 45,07 & 39,87 & 43,83 & 42,07 & 42,44 \\
Entre 2 y 3 SMMLV & 46,50 & 47,87 & 48,89 & 47,57 & 41,04 & 46,76 & 43,91 & 44,46 \\
Más de 3 SMMLV & 52,39 & 52,78 & 54,23 & 52,83 & 43,85 & 51,80 & 46,14 & 49,40 \\
Número de personas & & & & & & & & \\
Menos de 5 personas & 45,20 & 47,99 & 47,50 & 46,87 & 40,26 & 43,66 & 42,48 & 42,77 \\
Entre 5y 10 personas & 43,12 & 45,90 & 45,62 & 44,79 & 39,14 & 42,10 & 40,67 & 41,24 \\
Sin computador & 42,03 & 44,30 & 44,45 & 43,33 & 38,97 & 41,92 & 40,95 & 41,17 \\
Con Computador & 46,95 & 48,97 & 49,81 & 48,37 & 41,79 & 47,20 & 43,93 & 45,36 \\
Sin servicio de internet & 42,48 & 45,10 & 44,92 & 43,96 & 39,19 & 42,09 & 41,56 & 41,36 \\
Con Servicio de internet & 46,90 & 49,00 & 49,60 & 48,34 & 41,32 & 46,61 & 42,97 & 44,85 \\
Sin servicio de televisión cerrada & 42,43 & 45,64 & 44,70 & 44,18 & 38,95 & 41,56 & 40,34 & 40,94 \\
Con Servicio de televisión cerrada & 45,81 & 48,22 & 48,50 & 47,36 & 40,46 & 45,00 & 43,58 & 43,56 \\
\hline
\end{tabular}

Nota: El estrato socioeconómico bajo se integra con individuos con estrato 1 y 2; el estrato socioeconómico medio incluye los individuos en estrato 3 y 4; por último, el estrato socioeconómico alto contiene los individuos con estrato 5 y 6 .

Fuente: Esta investigación, con base en información del Instituto Colombiano para el Fomento de la Educación Superior.

Los resultados son similares cuando se analiza el nivel educativo de las madres. Se puede observar que los estudiantes con madres sin nivel educativo, en las regiones con mayor y menor nivel de desarrollo socioeconómico, tienen en promedio 11,11 y 7,39 puntos menos en relación con los estudiantes que tienen madres con nivel de Educación Superior. Del mismo modo, existe un diferencial en el puntaje promedio de los estudiantes a favor de las regiones desarrolladas, que es positivo y creciente a medida que se incrementa el nivel educativo de la 
madre, con una brecha de 2,74 puntos para los estudiantes con madres sin nivel educativo y llega a una diferencia de 6,25 puntos de alumnos que tienen madre con Educación Superior.

La actividad laboral que realiza el padre de familia es importante, debido al tiempo y conocimiento que puede dedicarles a sus hijos. Según las estimaciones realizadas durante el periodo 2008-2013, los estudiantes con padres de ocupación directiva obtienen mejor rendimiento en matemáticas, con un puntaje de 50,53 y 45,88 para las regiones con mayor y menor nivel de desarrollo socioeconómico respectivamente; les siguen los estudiantes con padres de ocupación laboral emprendedor, con 48,69 y 43,43 puntos, obreros con 45,17 y 42,78 puntos, otras actividades con 44,84 y 41,47 puntos y actividades del hogar con 44,04 y 40,21 puntos. Por otra parte, los estudiantes con padres asalariados obtienen 3,26 y 3,98 puntos más que los estudiantes con padres de ocupación independiente, en las regiones con mayor y menor nivel de desarrollo socioeconómico respectivamente.

Al comparar los puntajes por cada región y profesión del padre, se encuentra que los estudiantes de regiones desarrolladas obtienen mejores puntajes; los alumnos de padres emprendedores son los de mayor diferencia (5,26 puntos); les siguen, en su orden, los estudiantes con padres directivos (4,65 puntos), independientes $(4,40$ puntos), y estudiantes de padres que se dedican a realizar actividades del hogar (3,83 puntos).

Con respecto a las características de la vivienda y el hogar, el estrato socioeconómico, como se muestra en la Tabla 5, influye especialmente en la prueba de matemáticas. Los estudiantes de estrato alto ${ }^{6}$ obtienen en promedio 55,83 y 46,33 puntos para las regiones con mayor y menor nivel de desarrollo socioeconómico respectivamente, aproximadamente 11,52 y 4,53 puntos por encima de los estudiantes de estrato bajo; ${ }^{7}$ así mismo, en relación con los estudiantes de estrato medio, ${ }^{8}$ dicha diferencia desciende a 6,62 y 2,33 puntos respectivamente.

El ingreso mensual de los hogares es otra de las variables que influye en el rendimiento académico de los estudiantes. Los alumnos de hogares de ingresos mayores o iguales a 3 salarios mínimos obtienen un puntaje promedio de 52,83 puntos para las regiones desarrolladas y 49,40 puntos en las regiones con menor nivel de desarrollo. Los estudiantes con hogares que tienen menos de un salario mínimo obtienen en promedio 10,06 y 8,39 puntos menos que los estudiantes con hogares de ingresos superiores a 3 salarios, para las regiones con mayor y menor nivel de desarrollo socioeconómico respectivamente. Estos resultados muestran un efecto similar al obtenido por el estrato socioeconómico; a medida que se incrementa el ingreso mensual de los hogares, los estudiantes obtienen un mayor puntaje promedio.

\footnotetext{
6. Incluye los alumnos que pertenecen a los estratos 5 y 6 .

7. Incluye los alumnos que pertenecen a los estratos 1 y 2 .

8. Incluye los alumnos que pertenecen a los estratos 3 y 4 .
} 
Respecto al tamaño del hogar de los estudiantes, se encuentra que existe una diferencia entre estudiantes con grandes y pequeños hogares. Según las estimaciones, los estudiantes con hogares menores a 5 personas obtienen en promedio 2,08 y 1,53 puntos por encima de los hogares entre 5 y 10 personas para las regiones con mayor y menor nivel de desarrollo socioeconómico respectivamente. Por otra parte, los estudiantes de las regiones desarrolladas tienen en promedio 4,10 y 3,55 puntos más que los estudiantes de las regiones con menor nivel de desarrollo para los hogares con menos de 5 personas y los hogares entre 5 y 10 personas respectivamente.

Un componente importante que interviene en el desempeño académico de los estudiantes es el relacionado con las herramientas educativas. El computador, servicio de Internet y acceso a televisión cerrada son elementos importantes que facilitan el acceso y procesamiento de información, lo que trae consigo una mejor apropiación del conocimiento y, por ende, un mejor rendimiento académico.

Según las estimaciones realizadas, los estudiantes con computador en el hogar obtienen en promedio 5,04 y 4,19 más de puntos quelos estudiantes sin computador en casa, en las regiones con mayor y menor nivel de desarrollo socioeconómico respectivamente. Así mismo, al estudiar la brecha en el rendimiento entre regiones, se encuentra que los estudiantes que poseen computador en las regiones desarrolladas obtienen en promedio 48,37 puntos, 6\% (3,01 puntos) por encima de aquellos estudiantes que tienen computador y viven en las regiones con menor nivel de desarrollo.

Igualmente, los estudiantes con acceso a Internet tienden a obtener mayores puntajes en relación con los estudiantes sin acceso a este servicio. Esta diferencia es mayor en las regiones desarrolladas, en las que tener Internet en la vivienda implica obtener en promedio 4,38 puntos adicionales que aquellos estudiantes de las regiones con menor nivel de desarrollo. Por otro lado, el efecto del servicio de televisión cerrada sobre el rendimiento académico es significativo. Los estudiantes que tienen acceso al servicio de televisión cerrada obtienen un puntaje promedio 47,36 y 43,56 puntos, 3,18 y 2,62 puntos por encima de los estudiantes sin acceso a televisión para las regiones con mayor y menor nivel de desarrollo socioeconómico respectivamente. Al comparar el rendimiento entre ambas regiones, se determina que existe una brecha de $8 \%$ (3,80 puntos) y $7 \%$ (3,24 puntos) en favor de las regiones desarrolladas, para los estudiantes con y sin televisión.

\section{MODELO ECONOMÉTRICO}

Con el propósito de establecer cuáles son los principales determinantes del rendimiento académico en las regiones de mayor (Bogotá, Medellín y Santander) y menor (Chocó, Sucre y Vichada) nivel de desarrollo económico en Colombia, se utilizó el siguiente modelo lineal, inspirado en la función de producción educativa propuesta por Hanushek (1978): 
$g_{i p t}=\alpha+\beta^{T} X_{i p t}+\varphi^{T} Z_{p t}+e_{i p t} g_{i p t}=\alpha+\beta^{T} X_{i p t}+\varphi^{T} Z_{p t}+e_{i p t}$,

Donde $g_{i p t} g_{i p t}$ es el puntaje del individuo $i i$ que asiste al plantel $p p$ en el año $t$ $t ; X_{i p t} X_{i p t}$ es un vector de características de los individuos, que incluye, entre otras variables, el sexo, la edad, la educación de los padres y las condiciones socioeconómicas del hogar; $Z_{p t} Z_{p t}$ es un vector de características del plantel que incluye características como el tipo de jornada, la naturaleza de la institución y el carácter del bachillerato, y $e_{i p t} e_{i p t}$ es un término de error. La ecuación anterior se estimó al utilizar mínimos cuadrados ordinarios, a través de un modelo de combinación de corte transversal, pues se dispuso de información para seis años, 2008-2013.

Como se propone analizar el rendimiento académico en matemáticas, de acuerdo con los resultados de las pruebas Saber $11^{\circ}$, las estimaciones se realizaron al tener en cuenta el logaritmo del puntaje en matemáticas como variable independiente. Estos modelos se estimaron con utilización del software estadístico Stata 12.

La siguiente tabla muestra la incidencia de los distintos factores en el puntaje de matemáticas en las pruebas Saber $11^{\circ}$. Se presentan tres modelos: 1) el modelo general, que incluye estudiantes de las dos regiones analizadas; 2) el modelo para la región con mayor nivel de desarrollo y 3) el modelo para la región con menor nivel de desarrollo.

Tabla 6. Determinantes del rendimiento en matemáticas por regiones.

\begin{tabular}{|l|c|c|c|c|c|c|}
\hline \multicolumn{1}{|c|}{ Variables } & \multicolumn{2}{|c|}{ Modelo General } & Modelo Región Desarrollada & \multicolumn{2}{c|}{$\begin{array}{c}\text { Modelo Región } \\
\text { Subdesarrollada }\end{array}$} \\
\hline Características del Estudiante & Coef. & t-valor* & Coef. & t-valor* & Coef. & t-valor* \\
\hline Género y edad & \multicolumn{7}{|c}{} & & & \\
Edad & $-0,0044749$ & 80,02 & $-0,0043177$ & 75,6 & $-0,0075504$ & 27,69 \\
Hombre & 0,0570503 & 143,71 & 0,0588018 & 143,11 & 0,0366168 & 24,01 \\
Estudiante trabaja & $-0,0105037$ & 14,6 & $-0,0106848$ & 14,48 & $-0,0094254$ & 2,98 \\
Etnia & & & & & & \\
Negro & $-0,0723209$ & 55,11 & $-0,0515071$ & 24,9 & $-0,0790925$ & 42,64 \\
Indígena & $-0,0207191$ & 8,89 & $-0,0421279$ & 8,88 & $-0,014026$ & 4,88 \\
Raizal & $-0,0328991$ & 2,71 & $-0,0294477$ & 2,24 & -- & -- \\
Otras & $-0,0157667$ & 10,7 & $-0,0134704$ & 8,79 & $-0,039239$ & 7,39 \\
Prueba SABER & & & & & & \\
Presentó una vez & 0,0201999 & 20,8 & 0,0208811 & 21,31 & - & - \\
Presentó más de 2 veces & 0,0499381 & 20,43 & 0,0504353 & 20,57 & - & - \\
\hline
\end{tabular}




\begin{tabular}{|l|cccccc|}
\hline \multicolumn{1}{|c|}{ Características del Hogar } & \multicolumn{7}{c|}{ - } \\
Educación del Padre & \multicolumn{7}{c|}{} & - \\
Primaria & $-0,0061735$ & 7,82 & $-0,006906$ & 8,44 & - & - \\
Secundaria & 0,0054247 & 6,8 & 0,004354 & 5,31 & 0,0146615 & 7,51 \\
Terciaria & 0,040832 & 38,27 & 0,0403361 & 37,22 & 0,035998 & 8,2 \\
Superior & 0,0529417 & 49,09 & 0,0528061 & 48,61 & 0,0425242 & 11,69 \\
Educación de la Madre & & & & & & - \\
Primaria & 0,0099079 & 9,31 & 0,0110869 & 9,84 & - & - \\
Secundaria & 0,0260159 & 24,43 & 0,268817 & 23,95 & 0,0187302 & 9,65 \\
Terciaria & 0,0605114 & 49,37 & 0,0614541 & 48,1 & 0,0516518 & 12,95 \\
Superior & 0,0732071 & 57,33 & 0,0749388 & 56,21 & 0,0491308 & 13,07 \\
\hline
\end{tabular}

* Valor absoluto en la distribución t-student.

Fuente: Esta investigación.

Tabla 6. Determinantes del rendimiento en matemáticas por regiones (Continuación).

\begin{tabular}{|l|c|c|c|c|c|c|}
\hline \multirow{2}{*}{ Variables } & \multicolumn{2}{|c|}{ Modelo General } & \multicolumn{2}{c|}{$\begin{array}{c}\text { Modelo Región } \\
\text { Desarrollada }\end{array}$} & \multicolumn{2}{c|}{$\begin{array}{c}\text { Modelo Región } \\
\text { Subdesarrollada }\end{array}$} \\
\hline \multicolumn{1}{|c|}{ Características del Hogar } & Coef. & t-valor* & Coef. & t-valor* & Coef. & t-valor* \\
\hline $\begin{array}{l}\text { Ocupación del Padre } \\
\text { Emprendedor }\end{array}$ & 0,0095602 & 11,49 & 0,0104356 & 12,86 & -- & - \\
Directivo & $-0,0021792$ & 2,18 & -- & -- & -- & - \\
Asalariado & 0,0044317 & 5,5 & 0,0048651 & 6,26 & 0,011979 & 3,59 \\
Obrero & 0,0047296 & 7,47 & 0,0050492 & 8,24 & 0,0139671 & 4,64 \\
Independiente & 0,0112688 & 19,83 & 0,0120193 & 21,84 & 0,0060462 & 3,3 \\
Actividades del hogar & 0,0047804 & 2,72 & 0,0071125 & 3,9 & $-0,016708$ & 2,68 \\
Computador en casa & 0,0277501 & 48,94 & 0,0280369 & 48,17 & 0,0274673 & 11,22 \\
Servicio de internet en casa & 0,0016375 & 3,06 & 0,0015202 & 2,77 & 0,0056843 & 2,45 \\
Servicio de televisión cerrada & $-0,0023671$ & 4,97 & $-0,0037385$ & 7,54 & 0,0137453 & 7,95 \\
en casa & 0,0208527 & 47,43 & 0,0206141 & 45,21 & 0,0218838 & 13,09 \\
Residencia urbana & & & & & & \\
Estrato socioeconómico & 0,0243144 & 48,37 & 0,0240637 & 47,41 & 0,0369488 & 9,09 \\
Medio & 0,0635389 & 45,3 & 0,0642798 & 45,26 & 0,0408497 & 4,4 \\
Alto & & & & & & - \\
Ingresos & 0,0180513 & 32,23 & 0,0203991 & 34,51 & -- & - \\
Entre 1 y 2 SMMLV & 0,0292719 & 40,36 & 0,031956 & 42,58 & -- & -- \\
Entre 2 y 3 SMMLV & 0,0653121 & 73,9 & 0,0672281 & 74,21 & 0,0497442 & 12,11 \\
Más de 3 SMMLV & 0,0120197 & 25,83 & 0,0119137 & 24,43 & 0,0122176 & 7,82 \\
\hline Personas en la vivienda (<=5) & & & & & & \\
\hline Características del Colegio & & & & & & \\
\hline Tipo de Colegio & 0,0085617 & 18,62 & 0,0099346 & 20,8 & $-0,0034352$ & 1,99
\end{tabular}




\begin{tabular}{|l|ccccc|c|} 
Normalista & 0,0103526 & 7,27 & 0,0199749 & 11,88 & $-0,0218486$ & 7,56 \\
Colegio privado & 0,001516 & 2,71 & 0,0016625 & 2,91 & -- & -- \\
Paga pensión & -- & -- & -- & -- & 0,0130985 & 5,53 \\
Jornada & & & & & & \\
Completa & 0,0883862 & 107,01 & 0,0880553 & 103,73 & 0,0548135 & 9,09 \\
Mañana & 0,0636494 & 81,97 & 0,0634514 & 78,54 & 0,0705738 & 25,19 \\
Tarde & 0,0547666 & 65,18 & 0,0541871 & 62,26 & 0,0668447 & 20,92 \\
Región desarrollada & 0,027849 & 31,13 & -- & -- & -- & -- \\
\hline R2 & \multicolumn{2}{|c|}{$\mathbf{0 , 1 7 5 6}$} & $\mathbf{0 , 1 7 1 8}$ & $\mathbf{0 , 1 1 2 3}$ & \\
\hline Observaciones & $\mathbf{1 2 2 2 1 1 6}$ & $\mathbf{1 1 3 1 9 5 1}$ & $\mathbf{9 0 1 6 5}$ \\
\hline
\end{tabular}

* Valor absoluto en la distribución t-student.

Fuente: Esta investigación.

Los estudiantes de regiones con mayor nivel de desarrollo obtienen un 2,7\% más de puntaje en matemáticas que los estudiantes de regiones con menor nivel de desarrollo. Con respecto a las características propias de los estudiantes, el puntaje de los varones en matemáticas es 5,7\% más alto que el puntaje de las mujeres, resultado consistente con la evidencia empírica de países como Estados Unidos y otros países desarrollados. En las regiones con mayor nivel de desarrollo en Colombia, dicha diferencia se mantiene $(5,8 \%)$, mientras que en las regiones con menor nivel de desarrollo esta diferencia tiende a disminuir $(3,6 \%)$.

Los estudiantes más jóvenes obtienen, en promedio, puntajes más altos; un año adicional tiende a disminuir en $0,44 \%$ el puntaje en matemáticas. El efecto de la edad es mayor en regiones con menor nivel de desarrollo $(0,75 \%)$ frente a regiones con mayor nivel de desarrollo $(0,43 \%)$, aun después de controlar por el resto de características socioeconómicas del estudiante, así como por los factores del colegio. La situación laboral del estudiante también es importante; los estudiantes que trabajan obtienen un $1 \%$ menos de puntaje en matemáticas que los estudiantes que no lo hacen; en este aspecto, no se presentan grandes diferencias entre regiones desarrolladas y menos desarrolladas. Generalmente los individuos que trabajan presentan una mayor probabilidad de asistir a instituciones educativas de jornada nocturna o sabatina, cuyos puntajes son relativamente menores con respecto al resto de instituciones.

Los estudiantes de raza negra obtienen un 7,23\% menos puntaje en matemáticas que los estudiantes de otras razas; en las regiones con mayor nivel de desarrollo, dicha diferencia es de 5,15\%, mientras que en las regiones de menor nivel de desarrollo el coeficiente asciende al 8\%. Tanto los indígenas como los raizales también obtienen un menor puntaje que los estudiantes que no pertenecen a ninguna etnia, pero el efecto es mayor en las regiones con mayor nivel de desarrollo socioeconómico.

Los estudiantes con experiencia en la presentación de las pruebas Saber $11^{\circ}$ obtienen un mayor puntaje que los demás estudiantes, pero este efecto no es significativo en las regiones con menor nivel de desarrollo socioeconómico. 
En cuanto a las características familiares y del hogar de los estudiantes, se encontró que los hijos de padre con educación terciaria y superior, obtienen un $4 \%$ y $5,2 \%$ más de puntaje que los hijos con padres sin escolaridad. La educación de la madre, por su parte, presenta un mayor efecto estadístico sobre el rendimiento académico de los hijos que la educación del padre. Los hijos con madre de Educación Superior obtienen un 7,3\% más de puntaje que los hijos con madre sin escolaridad. En ambos casos, se observa que el impacto de la educación de los padres es mayor en las regiones con mayor nivel de desarrollo, aún después de controlar por las características del estudiante, del hogar y del colegio. Según Gaviria y Barrientos (2001), la conexión entre desempeño académico y educación de los padres puede darse a través de la calidad de los planteles educativos en los cuales matriculan a sus hijos, además de una mayor dotación de recursos tanto en la escuela como en el hogar (libros, computadores, viajes, etc.); esto es, padres con mejor nivel académico disponen de mayores recursos económicos, lo que les permite, a su vez, "comprar" una educación de mejor calidad para sus hijos.

El acceso a tecnologías se ha convertido en un aspecto importante para el logro académico; después de controlar por las características individuales, familiares y del colegio, se encontró que, en las regiones con mayor nivel de desarrollado, los estudiantes con acceso a computador en la vivienda obtienen un 2,8\% más de puntaje en matemáticas que el resto de estudiantes, similar al efecto en las regiones con menor nivel de desarrollo. Con respecto al acceso a Internet, el efecto es relativamente pequeño, siendo mayor el impacto en regiones con menor nivel de desarrollo.

Con respecto a las características económicas del hogar, los estudiantes de hogares con ingresos económicos superiores a tres salarios mínimos legales vigentes obtienen un 6,53\% más de puntaje en matemáticas que los estudiantes de hogares con menos de un salario mínimo. Aún después de controlar por las características del estudiante y el colegio, en las regiones con mayor nivel de desarrollo económico y social, los estudiantes de hogares con ingresos entre uno y dos salarios mínimos obtienen un 3,19\% más de puntaje que los hogares con menos de un salario; en los ingresos superiores a tres salarios mínimos, los estudiantes obtienen un 6,72\% más de puntaje que los estudiantes de menores ingresos. El efecto de los ingresos del hogar es significativo en las regiones con mayor nivel de desarrollo socioeconómico, mientras que en las regiones con menor nivel de desarrollo no se presenta significancia estadística.

Los estudiantes de viviendas con estrato socioeconómico bajo (estratos 1 y 2) de regiones con mayor nivel de desarrollo, obtienen un 6,42\% menos de puntaje que los estudiantes de estrato alto (estratos 5 y 6), y un 2,4\% menos que los estudiantes de estrato socioeconómico medio (estratos 3 y 4), aun después de controlar por las características del estudiante y el colegio. En las regiones con menor nivel de desarrollo, los estudiantes de viviendas con estrato socioeconómico bajo obtienen un 3,6\% menos de puntaje en matemáticas que los estudiantes de estrato medio y un $4 \%$ menos que los estudiantes de estrato alto. De nuevo se 
observa que el efecto de las características económicas del hogar es mayor en las regiones con condiciones económicas y sociales más favorables.

Los estudiantes que residen en la zona urbana obtienen un $2 \%$ más de puntaje en matemáticas que los estudiantes de la zona rural, aun después de controlar por las características del estudiante y el colegio. El efecto de la zona de residencia del estudiante es similar para las regiones con mayor y menor nivel de desarrollo del país, lo que se sustenta en la medida en que la zona rural, en la mayoría de las veces, limita el acceso a herramientas que permiten favorecer el aprendizaje estudiantil.

Finalmente, existe una conexión positiva entre las características de la institución educativa y el rendimiento académico de los estudiantes. Sorprende que el efecto del sector del colegio (público vs. privado) sea tan pequeño en el rendimiento de matemáticas. Aun después de controlar por las características individuales y familiares, los estudiantes de colegios privados de regiones desarrolladas obtienen un $0,16 \%$ más de puntaje que los estudiantes de colegios públicos; en las regiones con menor nivel de desarrollo, la variable indicativa del sector del colegio no resultó ser estadísticamente significativa.

Con respecto al carácter del colegio (académico, técnico, normalista), el efecto de los colegios técnicos es relativamente pequeño en las regiones con mayor y menor nivel de desarrollo socioeconómico, aun después de controlar por las características individuales y familiares del estudiante. El efecto de los colegios normalistas en las regiones con menor nivel de desarrollo es negativo, al indicar que los estudiantes de colegios normalistas de regiones menos desarrolladas obtienen un 2,1\% menos de puntaje que los estudiantes de colegios académicos. En las regiones con mayor nivel de desarrollo, el efecto de los colegios normalistas es positivo.

En cuanto a la jornada del colegio, es la variable (dentro de las características del colegio) con mayor impacto sobre el rendimiento académico de los estudiantes. Después de controlar por las características individuales y familiares, en las regiones con mayor nivel de desarrollo, los estudiantes de colegios con jornada completa obtienen un $8,8 \%$ más de puntaje que los estudiantes de colegios con jornada nocturna o sabatina; en las regiones con menor nivel de desarrollo, dicha diferencia es del 5,4\%. Con respecto a los colegios de jornada mañana y tarde, presentan un mayor efecto en las regiones con menor nivel de desarrollo; los estudiantes de la jornada nocturna o sabatina obtienen un 7\% y 6,6\% menos de puntaje que los estudiantes de la jornada mañana y tarde, respectivamente.

En cuanto a la bondad del ajuste del modelo, medido a través del R cuadrado, en el modelo general el coeficiente de determinación resultante fue de 0,1756 , que indica que la proporción de la variabilidad total del rendimiento en matemáticas se explica en un $17,56 \%$ por las variables contenidas en el modelo. En el caso del modelo para la región con mayor nivel de desarrollo económico, el $\mathrm{R}$ cuadrado fue de 0,1718, mientras que para la región con menor nivel descendió a 0,1123. Es evidente que las características del estudiante y de la escuela incluidas en los 
modelos, variables cuantitativas, ajustan y/o explican mejor el rendimiento en matemáticas en las regiones con mayor nivel de desarrollo.

En el Anexo 2, se repiten las estimaciones de los modelos para los cinco Departamentos estudiados y Bogotá (región con mayor nivel de desarrollado: Bogotá, Antioquía y Santander; región con menor nivel de desarrollo: Chocó, Sucre y Vichada). Los resultados de las estimaciones por Departamentos y Bogotá, confirman los hallazgos anteriores y sugieren, además, algunas diferencias en el efecto de las variables incluidas en los diferentes modelos.

Las diferencias en el rendimiento de matemáticas entre varones y mujeres son mayores en las regiones de mayor nivel de desarrollo económico; Bogotá registra la mayor diferencia $(6,25 \%)$ dentro de las regiones con mayor nivel de desarrollo, y Sucre $(4,47 \%)$ dentro de los Departamentos con menor nivel de desarrollo. El efecto de la edad sobre el rendimiento también es similar al obtenido previamente; en las regiones con menor nivel de desarrollo económico es donde mayor impacto registra. En cuanto a la situación laboral del estudiante, la variable estudiante trabaja es significativa en todas las regiones con mayor nivel de desarrollo, mientras que, en los Departamentos con menor nivel, solo en Sucre resultó ser significativa.

De otro lado, el efecto de la etnia a la que pertenecen los estudiantes es similar al obtenido en los modelos anteriores; la raza negra presenta mayores impactos en Departamentos como Sucre y Antioquia, mientras que en Chocó y Vichada los estudiantes indígenas alcanzan los rendimientos más bajos. Presentar la prueba antes tiene mayores efectos en los Departamentos de regiones con mayor nivel de desarrollo, especialmente sobre Santander.

El efecto de la educación de los padres presenta algunas diferencias con respecto a los resultados obtenidos previamente. Con respecto a la educación del padre, en Vichada, la variable no es significativa, mientras que en Chocó solo tiene efecto la Educación Superior. Por el contrario, los dos Departamentos y Bogotá, con mayor nivel de desarrollo, la educación del padre es significativa, y genera mayores impactos en Antioquia. En cuanto a la educación de la madre, presenta mayor impacto sobre el rendimiento académico de los estudiantes de regiones con menor desarrollo en comparación con la educación del padre.

Tener computador en casa es una variable que resulta ser significativa en todos los Departamentos y regiones analizados, y presenta mayor impacto en Departamentos como Sucre y Chocó; por el contrario, contar con servicio de Internet en la vivienda solo resulta ser significativo en los dos Departamentos y Bogotá, con mayor nivel de desarrollo.

En cuanto al estrato socioeconómico y los ingresos familiares, en Departamentos como Chocó y Vichada no presentaron significancia estadística, pues la distribución por ingresos y estrato se concentra altamente en los niveles más bajos, por lo que presenta poca variabilidad. Mientras que en Bogotá y los dos departamentos con mayor nivel de desarrollo, Santander y Antioquia registran los coeficientes más altos. 
Con respecto a las características del colegio, la jornada y el carácter de la institución son las variables que registran mayores impactos sobre el rendimiento de los estudiantes. Estas variables impactan en mayor medida en Bogotá y los departamentos de la región con mayor nivel de desarrollo, pues allí la presencia de mayores colegios privados garantiza una mayor variabilidad en cuanto a las características y dotaciones de los colegios, lo que les permite a los estudiantes y padres de familia tener mayores opciones de elección.

En cuanto a la bondad del ajuste del modelo, Bogotá y los departamentos de las regiones con mayor nivel de desarrollo presentan modelos mejor ajustados, pues es claro que las variables incluidas en los modelos registran mayores variabilidades en Bogotá y esos Departamentos, pues, además de contar con mayores poblaciones, las características familiares y del colegio son más evidentes en este tipo de regiones.

\section{CONCLUSIONES}

Con respecto a las características del estudiante, respecto al género, la diferencia en el rendimiento académico de matemáticas es mayor en regiones con menor nivel de desarrollo; los varones obtienen un puntaje mayor que las mujeres. El efecto de la edad sobre el rendimiento académico también es mayor en regiones con menor nivel de desarrollo.

La situación laboral del estudiante, a pesar de ser significativa, no genera impactos importantes sobre el rendimiento académico de los estudiantes de regiones con mayor y menor nivel de desarrollo socioeconómico.

Los estudiantes de raza negra alcanzan un menor puntaje en matemáticas que el resto de estudiantes; esta diferencia es mayor en las regiones con menor nivel de desarrollo. En el caso de los estudiantes de población indígena, la diferencia es mayor en regiones con mayor nivel de desarrollo económico.

Presentar la prueba con anterioridad tiene un efecto positivo en las regiones con mayor nivel de desarrollo; por el contrario, en regiones con menor nivel de desarrollo socioeconómico, dicha variable no presenta significancia estadística.

En cuanto a las características de la familia, tanto la educación del padre como la de la madre, generan un mayor nivel de impacto en las regiones con mayor nivel de desarrollo económico; a mayor nivel educativo de los padres, mayor puntaje obtienen los estudiantes. La educación de la madre presenta mayores impactos sobre el rendimiento académico de los estudiantes que la educación del padre, lo que ocurre en las regiones con mayor y menor nivel de desarrollo económico.

En cuanto a las dotaciones de la vivienda, el acceso a computador en casa presenta considerables impactos sobre el rendimiento académico de los estudiantes, 
en especial en las regiones con mayor nivel de desarrollo socioeconómico. Con respecto al acceso a Internet, el efecto sobre el rendimiento académico es relativamente menor, pero con mayor peso sobre las regiones con menor nivel de desarrollo.

Las características económicas del hogar, tales como el ingreso familiar y estrato socioeconómico de la vivienda, presentan mayores impactos sobre el rendimiento de estudiantes de regiones con mayor nivel de desarrollo socioeconómico, pues la distribución de hogares con condiciones económicas más favorables es mayor en las regiones con mayor nivel de desarrollo.

Con respecto a las características del colegio, la variable sector del colegio no presenta significancia estadística en las regiones con menor nivel de desarrollo socioeconómico, mientras que en las regiones con mayor nivel de desarrollo el efecto es significativo, pero relativamente pequeño. La jornada académica, es la característica del colegio que mayor efecto registra sobre el rendimiento académico; los colegios de la jornada de mañana y tarde tienen un mayor impacto sobre el rendimiento de estudiantes de regiones con menor nivel de desarrollo, mientras que los colegios de jornada completa tienen mayor impacto sobre el rendimiento académico de estudiantes de regiones con mayor nivel de desarrollo socioeconómico.

Por último, se concluye que la problemática acerca del rendimiento educativo y las pruebas Saber $11^{\circ}$ no se agota completamente, y menos cuando se estudian estas regiones, que presentan tantas desigualdades económicas y sociales. Esta es apenas una primera investigación, que debe servir de base para futuras investigaciones sobre el tema, que tiendan a contribuir a la solución del problema planteado.

Se recomienda, para futuras investigaciones, realizar este tipo de análisis en todos los ciclos de la educación, básica, media, superior, pues permitiría la caracterización global del Sistema de calidad educativa, para tornar más eficientes las decisiones tomadas hacia este sector. Del mismo modo, se pretende que este análisis sirva de base para la formulación de políticas diferenciadas, que propendan por un mayor logro académico en regiones con mayor y menor nivel de desarrollo socioeconómico. 


\section{ANEXOS}

\section{Anexo 1}

Tabla 7. Determinantes del rendimiento en matemáticas por departamentos. Regiones con mayor nivel de desarrollo socioeconómico.

\begin{tabular}{|c|c|c|c|c|c|c|}
\hline \multirow{2}{*}{\begin{tabular}{|c|} 
Variables \\
Características del Estudiante \\
\end{tabular}} & \multicolumn{2}{|c|}{ Modelo Bogotá } & \multicolumn{2}{|c|}{ Modelo Antioquia } & \multicolumn{2}{|c|}{ Modelo Santander } \\
\hline & Coef. & t-valor* & Coef. & t-valor* & Coef. & t-valor* \\
\hline \multicolumn{7}{|l|}{ Género y edad } \\
\hline Edad & $-0,0044177$ & $-50,31$ & $-0,0037948$ & $-43,05$ & $-0,0046208$ & $-31,58$ \\
\hline Hombre & 0,06254 & 109,87 & 0,0532603 & 77,61 & 0,0602738 & 52,74 \\
\hline Estudiante trabaja & $-0,0106042$ & $-9,96$ & $-0,0078027$ & $-6,27$ & $-0,0195036$ & $-10,74$ \\
\hline \multicolumn{7}{|l|}{ Etnia } \\
\hline Negro & $-0,0334336$ & $-7,69$ & $-0,0432181$ & $-17,79$ & -- & - \\
\hline Indígena & $-0,0180242$ & $-2,82$ & $-0,0670036$ & $-8,94$ & $-0,0473061$ & $-2,44$ \\
\hline Raizal & -- & -- & $-0,0556698$ & $-2,65$ & -- & - \\
\hline Otras & $-0,0162304$ & $-6,86$ & $-0,009098$ & $-4,04$ & -- & - \\
\hline \multicolumn{7}{|l|}{ Prueba SABER } \\
\hline Presentó una vez & 0,0132469 & 11,48 & $-0,0269983$ & $-8,43$ & 0,0285262 & 12,69 \\
\hline Presentó más de 2 vec. & 0,0348271 & 11,44 & $-0,0266999$ & $-2,41$ & 0,0517368 & 11,85 \\
\hline \multicolumn{7}{|l|}{ Características del Hogar } \\
\hline \multicolumn{7}{|l|}{ Educación del Padre } \\
\hline Primaria & $-0,0093935$ & $-12,3$ & $-0,0029852$ & $-2,66$ & $-0,0083029$ & $-5,72$ \\
\hline Secundaria & -- & -- & 0,009823 & 8,16 & -- & - \\
\hline Terciaria & 0,0344184 & 32,92 & 0,0504276 & 28,05 & 0,0343671 & 13,24 \\
\hline Superior & 0,0463526 & 42,12 & 0,0625413 & 34,05 & 0,0523627 & 20,71 \\
\hline \multicolumn{7}{|l|}{ Educación de la Madre } \\
\hline Primaria & 0,0099539 & 6,04 & -- & -- & 0,0175738 & 5,91 \\
\hline Secundaria & 0,0257225 & 16,33 & 0,0154754 & 17,84 & 0,0363153 & 12,45 \\
\hline Terciaria & 0,0571083 & 32,61 & 0,0548611 & 37,1 & 0,0722053 & 19,95 \\
\hline Superior & 0,073168 & 40,01 & 0,0609982 & 36,36 & 0,0848879 & 23,49 \\
\hline
\end{tabular}

* Valor absoluto en la distribución t-student.

Fuente: Elaboración propia. 
Tabla 7. Determinantes del rendimiento en matemáticas por departamentos. Regiones con mayor nivel de desarrollo socioeconómico. Continuación.

\begin{tabular}{|c|c|c|c|c|c|c|}
\hline \multirow{2}{*}{$\begin{array}{c}\text { Variables } \\
\text { Características del Hogar }\end{array}$} & \multicolumn{2}{|c|}{ Modelo Bogotá } & \multicolumn{2}{|c|}{ Modelo Antioquia } & \multicolumn{2}{|c|}{ Modelo Santander } \\
\hline & Coef. & t-valor* & Coef. & t-valor* & Coef. & t-valor* \\
\hline \multicolumn{7}{|l|}{ Ocupación del Padre } \\
\hline Emprendedor & 0,0043936 & 4,23 & 0,0082916 & 5,58 & 0,005125 & 2,41 \\
\hline Directivo & $-0,0042555$ & $-3,53$ & $-0,0051073$ & $-2,88$ & $-0,00607$ & $-2,01$ \\
\hline Asalariado & -- & -- & -- & -- & -- & - \\
\hline Obrero & 0,002004 & 2,47 & 0,0068901 & 7,3 & $-0,0057945$ & $-3,28$ \\
\hline Independiente & 0,0102884 & 14,09 & 0,0091418 & 10,62 & 0,0038444 & 2,65 \\
\hline Actividades del hogar & -- & -- & 0,0089126 & 3,19 & -- & - \\
\hline Computador en casa & 0,0277357 & 38,89 & 0,0262205 & 27,23 & 0,0277541 & 19,44 \\
\hline Servicio de internet en casa & $-0,0088976$ & $-12,7$ & 0,00403 & 4,39 & 0,0048148 & 3,52 \\
\hline Servicio de televisión cerrada en casa & -- & -- & 0,0021627 & 2,65 & -- & - \\
\hline Residencia urbana & 0,0216088 & 33,43 & 0,0129416 & 17,1 & 0,0252205 & 19,4 \\
\hline \multicolumn{7}{|l|}{ Estrato socioeconómico } \\
\hline Medio & 0,0237529 & 34,67 & 0,025995 & 30,25 & 0,028648 & 18,51 \\
\hline Alto & 0,0457571 & 21,02 & 0,0884196 & 40,02 & 0,083479 & 18,6 \\
\hline \multicolumn{7}{|l|}{ Ingresos } \\
\hline Entre 1 y $2 S M M L V$ & 0,0198138 & 20,3 & 0,0138995 & 15,71 & 0,0145228 & 9,69 \\
\hline Entre 2 y $3 S M M L V$ & 0,0293828 & 26,4 & 0,0238989 & 19,12 & 0,0259585 & 12,45 \\
\hline Más de 3 SMMLV & 0,0612779 & 48,19 & 0,0619786 & 38,12 & 0,0714817 & 27,74 \\
\hline Personas en la vivienda $(<=5)$ & 0,0129258 & 18,69 & 0,0126853 & 15,75 & 0,0120868 & 9,35 \\
\hline \multicolumn{7}{|l|}{ Características del Colegio } \\
\hline \multicolumn{7}{|l|}{ Tipo de Colegio } \\
\hline Técnico & 0,0050054 & 6,58 & 0,0114692 & 14,74 & 0,0112356 & 8,85 \\
\hline Normalista & -- & -- & 0,0179241 & 7,89 & 0,0414965 & 13,05 \\
\hline Colegio privado & -- & -- & -- & -- & $-0,0330499$ & $-20,34$ \\
\hline Paga pensión & $-0,0080857$ & $-9,24$ & 0,0114465 & 12,41 & -- & - \\
\hline \multicolumn{7}{|l|}{ Jornada } \\
\hline Completa & 0,0957837 & 70,11 & 0,0745413 & 56,6 & 0,0926748 & 40,68 \\
\hline Mañana & 0,0494215 & 38,74 & 0,0622827 & 50,52 & 0,077037 & 35,93 \\
\hline Tarde & 0,0434404 & 31,91 & 0,0567902 & 43,32 & 0,0663109 & 25,63 \\
\hline R2 & 0,165 & & 0,1612 & & 0,1773 & \\
\hline Observaciones & 55134 & & 427712 & & 152896 & \\
\hline
\end{tabular}

* Valor absoluto en la distribución t-student.

Fuente: Esta investigación. 
Tabla 8. Determinantes del rendimiento en matemáticas por departamentos. Regiones con menor nivel de desarrollo socioeconómico.

\begin{tabular}{|c|c|c|c|c|c|c|}
\hline \multirow{2}{*}{\begin{tabular}{|c|} 
Variables \\
Características del Estudiante
\end{tabular}} & \multicolumn{2}{|c|}{ Modelo Chocó } & \multicolumn{2}{|c|}{ Modelo Sucre } & \multicolumn{2}{|c|}{ Modelo Vichada } \\
\hline & Coef. & t-valor* & Coef. & t-valor* & Coef. & t-valor* \\
\hline \multicolumn{7}{|l|}{ Género y edad } \\
\hline Edad & $-0,0059396$ & $-11,47$ & $-0,0092498$ & $-29,21$ & $-0,0102009$ & $-7,58$ \\
\hline Hombre & 0,0204827 & 6,89 & 0,0447128 & 24,78 & 0,0441665 & 5,01 \\
\hline Estudiante trabaja & -- & -- & $-0,024116$ & $-6,17$ & -- & -- \\
\hline \multicolumn{7}{|l|}{ Etnia } \\
\hline Negro & $-0,0321914$ & $-7,94$ & $-0,0447417$ & $-10,75$ & -- & -- \\
\hline Indígena & $-0,1107521$ & $-7,94$ & -- & -- & $-0,0735552$ & $-5,92$ \\
\hline Raizal & -- & -- & -- & -- & -- & -- \\
\hline Otras & -- & -- & $-0,0317979$ & $-4,83$ & $-0,1086968$ & $-5,59$ \\
\hline \multicolumn{7}{|l|}{ Prueba SABER } \\
\hline Presentó una vez & $-0,0377164$ & $-2,45$ & -- & -- & -- & -- \\
\hline Presentó más de 2 veces & -- & -- & -- & -- & 0,1254026 & 2,37 \\
\hline \multicolumn{7}{|l|}{ Características del Hogar } \\
\hline \multicolumn{7}{|l|}{ Educación del Padre } \\
\hline Primaria & -- & -- & -- & -- & -- & -- \\
\hline Secundaria & -- & -- & 0,0181537 & 7,8 & -- & -- \\
\hline Terciaria & -- & -- & 0,0426009 & 8,35 & -- & -- \\
\hline Superior & 0,0210545 & 3,78 & 0,0572773 & 13,02 & -- & -- \\
\hline \multicolumn{7}{|l|}{ Educación de la Madre } \\
\hline Primaria & -- & -- & 0,0188501 & 8,18 & -- & -- \\
\hline Secundaria & 0,0151318 & 4,47 & -- & -- & 0,0385416 & 3,7 \\
\hline Terciaria & 0,0464856 & 6,08 & 0,0520513 & 11,09 & 0,0540586 & 2,64 \\
\hline Superior & 0,0407895 & 6,53 & 0,0598376 & 12,49 & 0,0801735 & 4 \\
\hline
\end{tabular}

* Valor absoluto en la distribución t-student.

Fuente: Esta investigación. 
Tabla 8. Determinantes del rendimiento en matemáticas por departamentos. Regiones con menor nivel de desarrollo socioeconómico. Continuación.

\begin{tabular}{|c|c|c|c|c|c|c|}
\hline \multirow{2}{*}{$\begin{array}{c}\text { Variables } \\
\text { Características del Hogar }\end{array}$} & \multicolumn{2}{|c|}{ Modelo Chocó } & \multicolumn{2}{|c|}{ Modelo Sucre } & \multicolumn{2}{|c|}{ Modelo Vichada } \\
\hline & Coef. & t-valor* & Coef. & t-valor* & Coef. & t-valor* \\
\hline \multicolumn{7}{|l|}{ Ocupación del Padre } \\
\hline Emprendedor & -- & -- & -- & -- & -- & -- \\
\hline Directivo & -- & -- & $-0,0179102$ & $-3,02$ & -- & -- \\
\hline Asalariado & -- & -- & -- & -- & -- & -- \\
\hline Obrero & 0,0145585 & 2,77 & 0,0066035 & 2,02 & -- & -- \\
\hline Independiente & -- & -- & -- & -- & -- & -- \\
\hline Actividades del hogar & -- & -- & -- & -- & -- & -- \\
\hline Computador en casa & 0,0291474 & 7,84 & 0,032567 & 11,96 & 0,0230224 & 2,17 \\
\hline Servicio de internet en casa & -- & -- & -- & -- & -- & -- \\
\hline Servicio de televisión cerrada en casa & -- & -- & 0,0147061 & 6,93 & -- & -- \\
\hline Residencia urbana & -- & -- & 0,03449 & 17,58 & 0,0368546 & 3,67 \\
\hline \multicolumn{7}{|l|}{ Estrato socioeconómico } \\
\hline Medio & 0,05825 & 5,44 & 0,0193821 & 4,28 & -- & -- \\
\hline Alto & -- & -- & 0,0470232 & 3,91 & -- & -- \\
\hline \multicolumn{7}{|l|}{ Ingresos } \\
\hline Entre 1 y $2 \mathrm{SMMLV}$ & -- & -- & 0,005081 & 2,22 & -- & -- \\
\hline Entre 2 y 3 SMMLV & -- & -- & 0,0172484 & 3,96 & -- & -- \\
\hline Más de 3 SMMLV & 0,0317819 & 4,4 & 0,0723859 & 12,87 & -- & -- \\
\hline Personas en la vivienda $(<=5)$ & 0,0145762 & 4,83 & 0,009375 & 5,05 & -- & -- \\
\hline \multicolumn{7}{|l|}{ Características del Colegio } \\
\hline \multicolumn{7}{|l|}{ Tipo de Colegio } \\
\hline Técnico & 0,0215249 & 6,11 & 0,0132653 & 6,24 & -- & -- \\
\hline Normalista & 0,0144363 & 2,74 & $-0,0104996$ & $-2,74$ & -- & -- \\
\hline Colegio privado & $-0,0279251$ & $-3,98$ & -- & -- & -- & -- \\
\hline Paga pensión & -- & -- & 0,0070183 & 2,3 & -- & -- \\
\hline \multicolumn{7}{|l|}{ Jornada } \\
\hline Completa & $-0,0190534$ & $-2,03$ & -- & -- & 0,0340216 & 2,46 \\
\hline Mañana & 0,0126481 & 3,25 & 0,028721 & 14,33 & -- & -- \\
\hline Tarde & & & -- & -- & -- & -- \\
\hline R2 & 0,0551 & & 0,1154 & & 0,1045 & \\
\hline Observaciones & 23503 & & 64102 & & 2560 & \\
\hline
\end{tabular}

* Valor absoluto en la distribución t-student.

Fuente: Esta investigación. 
Anexo 2.

Tabla 9. Estadísticas descriptivas.

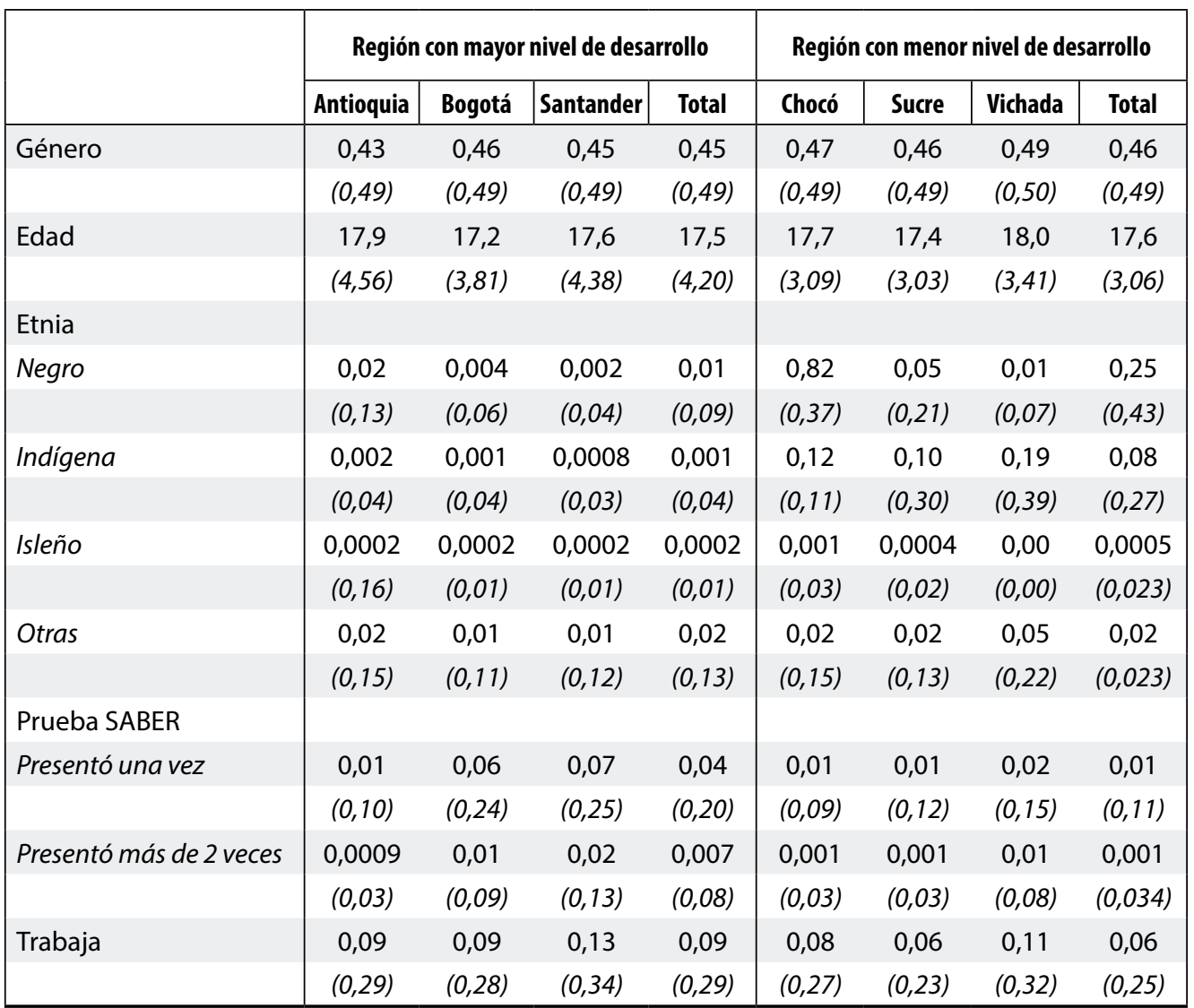

Fuente: Esta investigación, con base en información de Instituto Colombiano para el Fomento de la Educación Superior.

Tabla 9. Estadísticas descriptivas (continuación).

\begin{tabular}{|l|cccc|cccc|}
\hline Residencia Urbana & 0,68 & 0,72 & 0,64 & 0,70 & 0,70 & 0,60 & 0,62 & 0,62 \\
Educación del Padre & $(0,46)$ & $(0,44)$ & $(0,47)$ & $(0,45)$ & $(0,45)$ & $(0,48)$ & $(0,48)$ & $(0,48)$ \\
Ninguna & & & & & & & & \\
& 0,12 & 0,09 & 0,08 & 0,10 & 0,12 & 0,10 & 0,14 & 0,10 \\
Primaria & $(0,33)$ & $(0,28)$ & $(0,27)$ & $(0,30)$ & $(0,32)$ & $(0,30)$ & $(0,34)$ & $(0,31)$ \\
& 0,37 & 0,26 & 0,43 & 0,33 & 0,39 & 0,42 & 0,50 & 0,42 \\
Secundaria & $(0,48)$ & $(0,44)$ & $(0,49)$ & $(0,47)$ & $(0,48)$ & $(0,49)$ & $(0,50)$ & $(0,49)$ \\
& 0,35 & 0,40 & 0,32 & 0,37 & 0,34 & 0,34 & 0,25 & 0,34 \\
& $(0,47)$ & $(0,49)$ & $(0,46)$ & $(0,48)$ & $(0,47)$ & $(0,47)$ & $(0,43)$ & $(0,47)$ \\
\hline
\end{tabular}


Factores asociados al logro académico en regiones desarrolladas y en desarrollo de Colombia:

\begin{tabular}{|c|c|c|c|c|c|c|c|c|}
\hline \multirow[t]{2}{*}{ Técnica } & 0,06 & 0,10 & 0,06 & 0,08 & 0,35 & 0,04 & 0,03 & 0,04 \\
\hline & $(0,24)$ & $(0,30)$ & $(0,23)$ & $(0,27)$ & $(0,18)$ & $(0,19)$ & $(0,17)$ & $(0,19)$ \\
\hline \multirow[t]{2}{*}{ Superior } & 0,09 & 0,14 & 0,09 & 0,11 & 0,11 & 0,09 & 0,07 & 0,09 \\
\hline & $(0,27)$ & $(0,35)$ & $(0,28)$ & $(0,20)$ & $(0,32)$ & $(0,28)$ & $(0,25)$ & $(0,29)$ \\
\hline \multicolumn{9}{|c|}{ Educación de la Madre } \\
\hline \multirow[t]{2}{*}{ Ninguna } & 0,06 & 0,04 & 0,04 & 0,04 & 0,08 & 0,07 & 0,10 & 0,07 \\
\hline & $(0,23)$ & $(0,18)$ & $(0,20)$ & $(0,20)$ & $(0,27)$ & $(0,25)$ & $(0,29)$ & $(0,26)$ \\
\hline \multirow[t]{2}{*}{ Primaria } & 0,35 & 0,24 & 0,40 & 0,30 & 0,39 & 0,43 & 0,49 & 0,42 \\
\hline & $(0,47)$ & $(0,43)$ & $(0,48)$ & $(0,46)$ & $(0,48)$ & $(0,49)$ & $(0,50)$ & $(0,49)$ \\
\hline \multirow[t]{2}{*}{ Secundaria } & 0,42 & 0,45 & 0,39 & 0,43 & 0,37 & 0,37 & 0,29 & 0,37 \\
\hline & $(0,49)$ & $(0,49)$ & $(0,48)$ & $(0,49)$ & $(0,48)$ & $(0,48)$ & $(0,45)$ & $(0,48)$ \\
\hline \multirow[t]{2}{*}{ Técnica } & 0,09 & 0,13 & 0,07 & 0,11 & 0,04 & 0,05 & 0,05 & 0,04 \\
\hline & $(0,28)$ & $(0,33)$ & $(0,25)$ & $(0,30)$ & $(0,20)$ & $(0,22)$ & $(0,22)$ & $(0,21)$ \\
\hline \multirow[t]{2}{*}{ Superior } & 0,08 & 0,14 & 0,10 & 0,11 & 0,11 & 0,07 & 0,06 & 0,07 \\
\hline & $(0,27)$ & $(0,34)$ & $(0,29)$ & $(0,31)$ & $(0,31)$ & $(0,25)$ & $(0,23)$ & $(0,27)$ \\
\hline
\end{tabular}

Fuente: Esta investigación, con base en información de Instituto Colombiano para el Fomento de la Educación Superior.

Tabla 9. Estadísticas descriptivas (continuación).

\begin{tabular}{|c|c|c|c|c|c|c|c|c|}
\hline & \multicolumn{4}{|c|}{ Región con menor nivel de desarrollo } & \multicolumn{4}{|c|}{ Región con menor nivel de desarrollo } \\
\hline & Antioquia & Bogotá & Santander & Total & Chocó & Sucre & Vichada & Total \\
\hline \multicolumn{9}{|l|}{ Ocupación del Padre } \\
\hline \multirow[t]{2}{*}{ Emprendedor } & 0,06 & 0,10 & 0,10 & 0,09 & 0,04 & 0,05 & 0,08 & 0,05 \\
\hline & $(0,24)$ & $(0,30)$ & $(0,29)$ & $(0,28)$ & $(0,19)$ & $(0,22)$ & $(0,26)$ & $(0,22)$ \\
\hline \multirow[t]{2}{*}{ Directivo } & 0,04 & 0,07 & 0,04 & 0,06 & 0,03 & 0,03 & 0,03 & 0,02 \\
\hline & $(0,20)$ & $(0,26)$ & $(0,20)$ & $(0,23)$ & $(0,17)$ & $(0,16)$ & $(0,17)$ & $(0,16)$ \\
\hline \multirow[t]{2}{*}{ Asalariado } & 0,08 & 0,12 & 0,08 & 0,10 & 0,09 & 0,07 & 0,08 & 0,07 \\
\hline & $(0,26)$ & $(0,33)$ & $(0,27)$ & $(0,30)$ & $(0,28)$ & $(0,25)$ & $(0,27)$ & $(0,26)$ \\
\hline \multirow[t]{2}{*}{ Obrero } & 0,22 & 0,21 & 0,17 & 0,21 & 0,09 & 0,08 & 0,08 & 0,08 \\
\hline & $(0,42)$ & $(0,41)$ & $(0,38)$ & $(0,41)$ & $(0,28)$ & $(0,27)$ & $(0,27)$ & $(0,27)$ \\
\hline \multirow[t]{2}{*}{ Independiente } & 0,31 & 0,29 & 0,41 & 0,31 & 0,50 & 0,59 & 0,54 & 0,56 \\
\hline & $(0,46)$ & $(0,45)$ & $(0,49)$ & $(0,46)$ & $(0,50)$ & $(0,49)$ & $(0,49)$ & $(0,49)$ \\
\hline \multirow[t]{2}{*}{ Actividades del hogar } & 0,02 & 0,01 & 0,01 & 0,01 & 0,02 & 0,01 & 0,02 & 0,01 \\
\hline & $(0,12)$ & $(0,10)$ & $(0,10)$ & $(0,11)$ & $(0,13)$ & $(0,12)$ & $(0,13)$ & $(0,12)$ \\
\hline \multirow[t]{2}{*}{ Otras } & 0,26 & 0,18 & 0,17 & 0,20 & 0,23 & 0,16 & 0,16 & 0,18 \\
\hline & $(0,43)$ & $(0,38)$ & $(0,38)$ & $(0,40)$ & $(0,42)$ & $(0,36)$ & $(0,36)$ & $(0,38)$ \\
\hline
\end{tabular}




\begin{tabular}{|c|c|c|c|c|c|c|c|c|}
\hline \multicolumn{9}{|c|}{ Estrato socioeconómico } \\
\hline \multirow[t]{2}{*}{ Bajo } & 0,65 & 0,56 & 0,68 & 0,61 & 0,97 & 0,94 & 0,92 & 0,94 \\
\hline & $(0,47)$ & $(0,49)$ & $(0,46)$ & $(0,48)$ & $(0,16)$ & $(0,24)$ & $(0,27)$ & $(0,22)$ \\
\hline \multirow[t]{2}{*}{ Medio } & 0,31 & 0,42 & 0,29 & 0,36 & 0,02 & 0,05 & 0,02 & 0,05 \\
\hline & $(0,46)$ & $(0,49)$ & $(0,45)$ & $(0,48)$ & $(0,14)$ & $(0,22)$ & $(0,13)$ & $(0,20)$ \\
\hline \multirow[t]{2}{*}{ Alto } & 0,04 & 0,02 & 0,02 & 0,03 & 0,01 & 0,01 & 0,06 & 0,01 \\
\hline & $(0,18)$ & $(0,14)$ & $(0,14)$ & $(0,16)$ & $(0,07)$ & $(0,07)$ & $(0,23)$ & $(0,08)$ \\
\hline
\end{tabular}

Nota: Del estrato socioeconómico bajo hacen parte los individuos con estrato 1 y 2; estrato socioeconómico medio incluye los individuos en estrato 3 y 4; por último, el estrato socioeconómico alto contiene los individuos con estrato 5 y 6 .

Fuente: Esta investigación, con base en información de Instituto Colombiano para el Fomento de la Educación Superior.

Tabla 9. Estadísticas descriptivas (continuación).

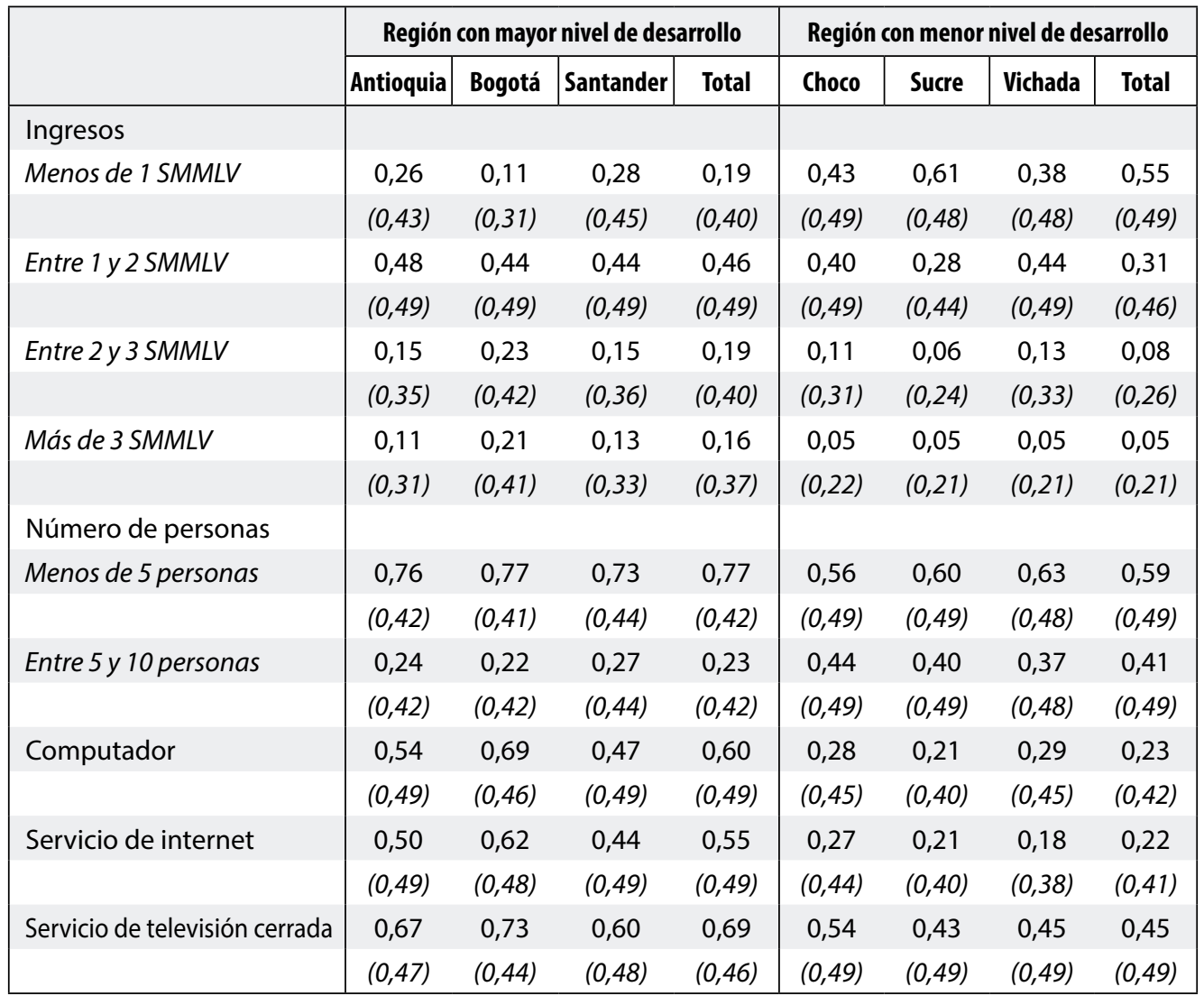

Fuente: Esta investigación, con base en información de Instituto Colombiano para el Fomento de la Educación Superior. 


\section{REFERENCIAS BIBLIOGRÁFICAS}

Angrist, J. D., y A. B. Krueger. 1991. Does compulsory schooling attendance affect schooling and earnings? Quarterly Journal of Economics, 4, $\mathrm{n}^{\circ} 106$ (nov.):979-1014.

Barrientos, J. H. 2008. Calidad de la educación pública y logro académico en Medellín 2004-2006. Una aproximación por regresión intercuartil. Lecturas de Economía 68: 121-144.

Caro, B. L. 2000. Factores asociados al logro académico de los alumnos de $3^{\circ}$ y $5^{\circ}$ de primaria de Bogotá. Coyuntura Social - Fedesarrollo 22 (mayo):65-80.

Cerquera Losada, O. H. (2011). Determinantes de la Calidad de la Educación en Neiva. Programa Jóvenes Investigadores, 192.

Chica Gómez, S. M.; D. M. Galvis Gutiérrez y A. Ramírez Hassan. 2010. Determinantes del Rendimiento Académico en Colombia. Revista Universidad Eafit 46, n 160:48-72.

Coleman, J. S. 1966. Equality of educational opportunity. Washington, US Government Printing Office.

Cox, D., y E. Jiménez. 1991. The Relative Effectiveness of Private and Public Schools: Evidence from Two Developing Countries. The Journal of Development Economics 34 (oct.):99-121.

Darling-Hammond, L. 2000. Teacher quality and student achievement: a review of state policy evidence. Education Policy Analysis Archives 8, $\mathrm{n}^{\circ} 1$ (enero).

Eskeland, G. S., y D. Filmer. 2002. Autonomy, participation, and learning in Argentine schools: Findings, and Their Implications for Decentralization. Policy Research Working Paper 2766.

Fuchs, T., y L. Woessmann. 2005. What accounts for international differences in student performance? are-examinationusing PISA data. Empirical Economics 2, n 32 (mayo):433-464.

Gaviria, A., y J. H. Barrientos. 2001. Determinantes de la calidad de la educación en Colombia. Arvhivos de Economía 159.

Hanushek, E. A. (1986). The economics of Schooling. Journal of Economic Literature 24, nº 3 (sept.):11411171.

Hepp K., P.; S. E. Hinostroza, M. E. Laval y F. L. Rehbein. 2004. Technology in schools: education, ICT, and the knowledge society. World Bank 94.

Kerlinger, F. 1983. Investigación del comportamiento. Técnicas y metodología (2ª edición). México: Editorial Interamericana.

King, E., y B. Özler. 2000. What's decentralization got to do with learning? endogenous school quality and student performance in Nicaragua. World Bank 58.

López, S. F. 2010. El efecto colegio en Colombia: tres décadas de estudio. Equidad \& Desarrollo 14 (jul-dic.): 85-101.

Mina Calvo, A. 2004. Factores asociados al logro educativo a nivel Municipal. Documento CEDE 15 (marzo).

Piñeros, L. J., y A. Rodríguez. 1998. Los insumos escolares en la educación secundaria y su efecto sobre el rendimiento académico de los estudiantes: un estudio en Colombia. LCSHD Paper Series, 36 (diciembre).

Sanders, W. L., J. C. Rivers. 1996. Cumulative and residual effects of teachers on future student academic achievement. Research Progress Report (noviembre).

Vegas, E., y J. Petrow. 2008. Incrementar el aprendizaje estudiantil en América Latina. (M. T. Barajas S., Ed., e I. Caviedes Hoyos, Trad.) Washington: Banco Mundial.

Wolff, L., J. C. Navarro y P. González. 2005. Private education and public policy in Latin America. Washington: Partnership for Educational Revitalization in the Americas. 